\title{
Robust Portfolio Optimization with Derivative Insurance Guarantees
}

\author{
Steve Zymler*, Berç Rustem and Daniel Kuhn \\ Department of Computing \\ Imperial College of Science, Technology and Medicine \\ 180 Queen's Gate, London SW7 2AZ, UK.
}

April 21, 2010

\begin{abstract}
Robust portfolio optimization aims to maximize the worst-case portfolio return given that the asset returns are allowed to vary within a prescribed uncertainty set. If the uncertainty set is not too large, the resulting portfolio performs well under normal market conditions. However, its performance may substantially degrade in the presence of market crashes, that is, if the asset returns materialize far outside of the uncertainty set. We propose a novel robust optimization model for designing portfolios that include European-style options. This model trades off weak and strong guarantees on the worst-case portfolio return. The weak guarantee applies as long as the asset returns are realized within the prescribed uncertainty set, while the strong guarantee applies for all possible asset returns. The resulting model constitutes a convex second-order cone program, which is amenable to efficient numerical solution procedures. We evaluate the model using simulated and empirical backtests and analyze the impact of the insurance guarantees on the portfolio performance.
\end{abstract}

Key words: robust optimization, portfolio optimization, portfolio insurance, second-order cone programming.

\section{Introduction}

Investors face the challenging problem of how to distribute their current wealth over a set of available assets, such as stocks, bonds, and derivatives, with the goal to earn the highest possible future wealth. One of the first mathematical models for this problem was formulated by Harry Markowitz [32]. In his Nobel prize-winning work, he observed that a rational investor does not aim solely at maximizing the expected return of an investment, but also at minimizing its risk. In the Markowitz model, the risk of a portfolio is measured by the variance of the portfolio return. A practical advantage of the Markowitz model is that it reduces to a convex quadratic program, which can be solved efficiently.

\footnotetext{
*Corresponding author: sz02@doc.ic.ac.uk
} 
Although the Markowitz model has triggered a tremendous amount of research activities in the field of finance, it has serious disadvantages which have discouraged practitioners from using it. The main problem is that the means and covariances of the asset returns, which are important inputs to the model, have to be estimated from noisy data. Hence, these estimates are not accurate. In fact, it is fundamentally impossible to estimate the mean returns with statistical methods to within workable precision, a phenomenon which is sometimes referred to as mean blur [29, 34]. Unfortunately, the mean-variance model is very sensitive to the distributional input parameters. As a result, the model amplifies any estimation errors, yielding extreme portfolios which perform badly in out-of-sample tests [16, 12, 36, 18].

Many attempts have been undertaken to ease this amplification of estimation errors. Black and Litterman [10] suggest Bayesian estimation of the means and covariances using the market portfolio as a prior. Jagannathan and Ma [26] as well as Chopra [14] impose portfolio constraints in order to guide the optimization process towards more intuitive and diversified portfolios. Chopra et al. [15] use a James-Steiner estimator for the means which tilts the optimal allocations towards the minimum-variance portfolio, while DeMiguel et al. [18] employ robust estimators.

In recent years, robust optimization has received considerable attention. Robust optimization is a powerful modelling paradigm for decision problems subject to non-stochastic data uncertainty [6]. The uncertain problem parameters are assumed to be unknown but confined to an uncertainty set, which reflects the decision maker's uncertainty about the parameters. Robust optimization models aim to find the best decision in view of the worst-case parameter values within these sets. Ben-Tal and Nemirovski [7] propose a robust optimization model to immunize a portfolio against the uncertainty in the asset returns. They show that when the asset returns can vary within an ellipsoidal uncertainty set determined through their means and covariances, the resulting optimization problem is reminiscent of the Markowitz model. This robust portfolio selection model still assumes that the distributional input parameters are known precisely. Therefore, it suffers from the same shortcomings as the Markowitz model.

Robust portfolio optimization can also be used to immunize a portfolio against the uncertainty in the distributional input parameters. Goldfarb and Iyengar [22] use statistical methods for constructing uncertainty sets for factor models of the asset returns and show that their robust portfolio problem can be reformulated as a second-order cone program. Tütüncü and Koenig [41] propose a model with box uncertainty sets for the means and covariances and show that the arising model can be reduced to a smooth saddle-point problem subject to semidefinite constraints. Rustem and Howe [39] describe algorithms to solve general continuous and discrete minimax problems and present several applications of worst-case optimization for risk management. Rustem et al. [38] propose a model that optimizes the worst-case portfolio return under rival risk and return forecasts in a discrete minimax setting. El Ghaoui et al. [20] show that the worst-case Valueat-Risk under partial information on the moments can be formulated as a semidefinite program. Ben-Tal et al. [5] as well as Bertsimas and Pachamanova [9] suggest robust portfolio models in a multi-period setting. A recent survey of applications of robust portfolio optimization is provided in the monograph [21]. Robust portfolios of this kind are relatively insensitive to the distributional input parameters and typically outperform classical Markowitz portfolios [13].

Robust portfolios exhibit a non-inferiority property [38]: whenever the asset returns are realized within the prescribed uncertainty set, the realized portfolio return will be greater than or equal to the calculated worst-case portfolio return. Note that this property may fail to hold when the asset returns happen to fall outside of the uncertainty set. 
In this sense, the non-inferiority property only offers a weak guarantee. When a rare event (such as a market crash) occurs, the asset returns can materialize far beyond the uncertainty set, and hence the robust portfolio will remain unprotected. A straightforward way to overcome this problem is to enlarge the uncertainty set to cover also the most extreme events. However, this can lead to robust portfolios that are too conservative and perform poorly under normal market conditions.

In this paper we will use portfolio insurance to hedge against rare events which are not captured by a reasonably sized uncertainty set. Classical portfolio insurance is a well studied topic in finance. The idea is to enrich a portfolio with specific derivative products in order to obtain a deterministic lower bound on the portfolio return. The insurance holds for all possible realizations of the asset returns and can therefore be qualified as a strong guarantee. Numerous studies have investigated the integration of options in portfolio optimization models. Ahn et al. [1] minimize the Value-at-Risk of a portfolio consisting of a single stock and a put option by controlling the portfolio weights and the option strike price. Dert and Oldenkamp [19] propose a model that maximizes the expected return of a portfolio consisting of a single index stock and several European options while guaranteeing a maximum loss. Howe et al. [24] introduce a risk management strategy for the writer of a European call option based on minimax using box uncertainty. Lutgens et al. [30] propose a robust optimization model for option hedging using ellipsoidal uncertainty sets. They formulate their model as a second-order cone program which may have, in the worst-case, an exponential number of conic constraints.

This paper combines robust portfolio optimization and classical portfolio insurance with the objective of providing two layers of guarantees. The weak non-inferiority guarantee applies as long as the returns are realized within the uncertainty set, while the strong portfolio insurance guarantee also covers cases in which the returns are realized outside of the uncertainty set. The ideas set out in this paper are related to the concept of Comprehensive Robustness proposed by Ben-Tal et al. [4]. Comprehensive Robustness aims to control the deterioration in performance when the uncertainties materialize outside of the uncertainty set. Our work establishes the relationship between offering guarantees beyond the uncertainty set and portfolio insurance. Indeed, we will show that in order to control the deterioration in portfolio return, our model will allocate wealth in put and call options. The premia of these options will determine the cost to satisfy the guarantee levels. Our contributions can be summarized as follows:

(1) We extend the existing robust portfolio optimization models to include options as well as stocks. Because option returns are convex piece-wise linear functions of the underlying stock returns, options cannot be treated as additional stocks, and the use of an ellipsoidal uncertainty set is no longer adequate. Under a no short-sales restriction on the options, we demonstrate how our model can be reformulated as a convex second-order cone program that scales gracefully with the number of stocks and options. We also show that our model implicitly minimizes a coherent risk measure [3]. Coherency is a desirable property from a risk management viewpoint.

(2) We describe how the options in the portfolio can be used to obtain additional strong guarantees on the worst-case portfolio return even when the stock returns are realized outside of the uncertainty set. We show that the arising Insured Robust Portfolio Optimization model trades off the guarantees provided through the non-inferiority property and the derivative insurance strategy. Using conic duality, we reformulate this model as a tractable second-order cone program.

(3) We perform a variety of numerical experiments using simulated as well as real market 
data. In our simulated tests we illustrate the tradeoff between the non-inferiority guarantee and the strong insurance guarantee. We also evaluate the performance of the Insured Robust Portfolio Optimization model under "normal" market conditions, in which the asset prices are governed by geometric Brownian motions, as well as in a market environment in which the prices experience significant downward jumps. The impact of the insurance guarantees on the portfolio performance is also analyzed using real market prices.

The rest of the paper is organized as follows. In Section 2 we review robust portfolio optimization and elaborate on the non-inferiority guarantee. In Section 3 we show how a portfolio that contains options can be modelled in a robust optimization framework and how strong insurance guarantees can be imposed on the worst-case portfolio return. We also demonstrate how the resulting model can be formulated as a tractable secondorder cone program. In Section 4 we report on numerical tests in which we compare the insured robust model with the standard robust model as well as the classical meanvariance model. We run simulated as well as empirical backtests. Conclusions are drawn in Section 5, and a notational reference table is provided in Appendix A.1.

\section{Robust Portfolio Optimization}

Consider a market consisting of $n$ stocks. Moreover, denote the current time as $t=0$ and the end of investment horizon as $t=T$. A portfolio is completely characterized by a vector of weights $\boldsymbol{w} \in \mathbb{R}^{n}$, whose elements add up to 1 . The component $w_{i}$ denotes the percentage of total wealth which is invested in the $i$ th stock at time $t=0$. Furthermore, let $\tilde{\boldsymbol{r}}$ denote the random vector of total stock returns over the investment horizon, which takes values in $\mathbb{R}_{+}^{n}$. By definition, the investor will receive $\tilde{r}_{i}$ dollars at time $T$ for every dollar invested in stock $i$ at time 0 . We will always denote random variables by symbols with tildes, while their realizations are denoted by the same symbols without tildes. The return vector $\tilde{\boldsymbol{r}}$ is representable as

$$
\tilde{\boldsymbol{r}}=\boldsymbol{\mu}+\tilde{\boldsymbol{\epsilon}}
$$

where $\boldsymbol{\mu}=\mathbb{E}[\tilde{\boldsymbol{r}}] \in \mathbb{R}_{+}^{n}$ denotes the vector of mean returns and $\tilde{\boldsymbol{\epsilon}}=\tilde{\boldsymbol{r}}-\mathbb{E}[\tilde{\boldsymbol{r}}]$ stands for the vector of residual returns. We assume that $\operatorname{Cov}[\tilde{\boldsymbol{r}}]=\mathbb{E}\left[\tilde{\boldsymbol{\epsilon}} \tilde{\boldsymbol{\epsilon}}^{T}\right]=\boldsymbol{\Sigma} \in \mathbb{R}^{n \times n}$ is strictly positive definite. The return $\tilde{r}_{p}$ on some portfolio $\boldsymbol{w}$ is given by

$$
\tilde{r}_{p}=\boldsymbol{w}^{T} \tilde{\boldsymbol{r}}=\boldsymbol{w}^{T} \boldsymbol{\mu}+\boldsymbol{w}^{T} \tilde{\boldsymbol{\epsilon}}
$$

Markowitz suggested to determine an optimal tradeoff between the expected return $\mathbb{E}\left[\tilde{r}_{p}\right]$ and the risk $\operatorname{Var}\left[\tilde{r}_{p}\right]$ of the portfolio [32]. The optimal portfolio can thus be found by solving the following convex quadratic program

$$
\max _{\boldsymbol{w} \in \mathbb{R}^{n}}\left\{\boldsymbol{w}^{T} \boldsymbol{\mu}-\lambda \boldsymbol{w}^{T} \boldsymbol{\Sigma} \boldsymbol{w} \mid \boldsymbol{w}^{T} \mathbf{1}=1, \boldsymbol{l} \leq \boldsymbol{w} \leq \boldsymbol{u}\right\},
$$

where the parameter $\lambda$ characterizes the investor's risk-aversion, the constant vectors $\boldsymbol{l}, \boldsymbol{u} \in \mathbb{R}^{n}$ are used to model portfolio constraints, and $\mathbf{1} \in \mathbb{R}^{n}$ denotes a vector of $1 \mathrm{~s}$.

\subsection{Basic Model}

Robust optimization offers a different interpretation of the classical Markowitz problem. Ben-Tal and Nemirovski [7] argue that the investor wishes to maximize the portfolio 
return and thus attempts to solve the uncertain linear program

$$
\max _{\boldsymbol{w} \in \mathbb{R}^{n}}\left\{\boldsymbol{w}^{T} \tilde{\boldsymbol{r}} \mid \boldsymbol{w}^{T} \mathbf{1}=1, \boldsymbol{l} \leq \boldsymbol{w} \leq \boldsymbol{u}\right\} .
$$

However, this problem is not well-defined. It constitutes a whole family of linear programs. In fact, for each return realisation we obtain a different optimal solution. In order to disambiguate the investment decisions, robust optimization adopts a worst-case perspective. In this modelling framework, the return vector $\tilde{\boldsymbol{r}}$ remains unknown, but it is believed to materialize within an uncertainty set $\Theta_{r}$. To immunize the portfolio against the inherent uncertainty in $\tilde{\boldsymbol{r}}$, we maximize the worst-case portfolio return, where the worst-case is calculated with respect to all asset returns in $\Theta_{r}$. This can be formalized as a max-min problem

$$
\max _{\boldsymbol{w} \in \mathbb{R}^{n}}\left\{\min _{\boldsymbol{r} \in \Theta_{\boldsymbol{r}}} \boldsymbol{w}^{T} \boldsymbol{r} \mid \boldsymbol{w}^{T} \mathbf{1}=1, \boldsymbol{l} \leq \boldsymbol{w} \leq \boldsymbol{u}\right\} .
$$

The objective function in (3) represents the worst-case portfolio return should $\tilde{\boldsymbol{r}}$ be realized within $\Theta_{r}$. Note that this quantity depends in a non-trivial way on the portfolio vector $\boldsymbol{w}$.

There are multiple ways to specify $\Theta_{r}$. A natural choice is to use an ellipsoidal uncertainty set

$$
\Theta_{\boldsymbol{r}}=\left\{\boldsymbol{r}:(\boldsymbol{r}-\boldsymbol{\mu})^{T} \boldsymbol{\Sigma}^{-1}(\boldsymbol{r}-\boldsymbol{\mu}) \leq \delta^{2}\right\} .
$$

As shown in an influential paper by El Ghaoui et al. [20], when $\tilde{\boldsymbol{r}}$ has finite second-order moments, then, the choice

$$
\delta=\sqrt{\frac{p}{1-p}} \text { for } p \in[0,1) \quad \text { and } \quad \delta=+\infty \text { for } p=1
$$

implies the following probabilistic guarantee for any portfolio $\boldsymbol{w}$ :

$$
\mathbb{P}\left\{\boldsymbol{w}^{T} \tilde{\boldsymbol{r}} \geq \min _{\boldsymbol{r} \in \Theta_{\boldsymbol{r}}} \boldsymbol{w}^{T} \boldsymbol{r}\right\} \geq p
$$

The investor controls the size of the uncertainty set by choosing the parameter $p$. For $p$ close to 0 , the ellipsoid shrinks to $\{\boldsymbol{\mu}\}$, and therefore the investor is only concerned about the average performance of the portfolio. When $p$ is close to 1 , the ellipsoid becomes very large, which implies that the investor wants to safeguard against a large set of possible return outcomes. Thus, the choice of uncertainty set size depends on the risk attitude of the investor.

It is shown in [7] that for ellipsoidal uncertainty sets of the type (4), problem (3) reduces to a convex second-order cone program [27].

$$
\max _{\boldsymbol{w} \in \mathbb{R}^{n}}\left\{\boldsymbol{w}^{T} \boldsymbol{\mu}-\delta\left\|\boldsymbol{\Sigma}^{1 / 2} \boldsymbol{w}\right\|_{2} \mid \boldsymbol{w}^{T} \mathbf{1}=1, \boldsymbol{l} \leq \boldsymbol{w} \leq \boldsymbol{u}\right\}
$$

Note that (7) is very similar to the classical Markowitz model (2). The main difference is that the standard deviation $\left\|\boldsymbol{\Sigma}^{1 / 2} \boldsymbol{w}\right\|_{2}=\sqrt{\boldsymbol{w}^{T} \Sigma \boldsymbol{w}}$ replaces the variance. The parameter $\delta$ is the analogue of $\lambda$, which determines the risk-return tradeoff. It can be shown that (2) and (7) are equivalent problems in the sense that for every $\lambda$ there is some $\delta$ for which the two problems have the same optimal solution. 


\subsection{Parameter Uncertainty}

In the Introduction we outlined the shortcomings of the Markowitz model, which carry over to the equivalent mean-standard deviation model (7): both models are highly sensitive to the distributional input parameters $(\boldsymbol{\mu}, \boldsymbol{\Sigma})$. These parameters, in turn, are difficult to estimate from noisy historical data. The optimization problems (2) and (7) amplify these estimation errors, yielding extreme portfolios that perform poorly in out-of-sample tests. It turns out that robust optimization can also be used to immunize the portfolio against uncertainties in $\boldsymbol{\mu}$ and $\boldsymbol{\Sigma}$. The starting point of such a robust approach is to assume that the true parameter values are unknown but contained in some uncertainty sets which reflect the investor's confidence in the parameter estimates.

Assume that the true (but unobservable) mean vector $\boldsymbol{\mu} \in \mathbb{R}^{n}$ is known to belong to a set $\Theta_{\boldsymbol{\mu}}$, and the true covariance matrix $\boldsymbol{\Sigma} \in \mathbb{R}^{n \times n}$ is known to belong to a set $\Theta_{\boldsymbol{\Sigma}}$. Robust portfolio optimization aims to find portfolios that perform well under worst-case values of $\boldsymbol{\mu}$ and $\boldsymbol{\Sigma}$ within the corresponding uncertainty sets. The parameter robust generalization of problem (7) can thus be formulated as

$$
\max _{\boldsymbol{w} \in \mathbb{R}^{n}}\left\{\min _{\boldsymbol{\mu} \in \Theta_{\mu}} \boldsymbol{w}^{T} \boldsymbol{\mu}-\delta \max _{\boldsymbol{\Sigma} \in \Theta_{\boldsymbol{\Sigma}}}\left\|\boldsymbol{\Sigma}^{1 / 2} \boldsymbol{w}\right\|_{2} \mid \boldsymbol{w}^{T} \mathbf{1}=1, \boldsymbol{l} \leq \boldsymbol{w} \leq \boldsymbol{u}\right\} .
$$

There are multiple ways to specify the new uncertainty sets $\Theta_{\boldsymbol{\mu}}$ and $\Theta_{\boldsymbol{\Sigma}}$. Let $\hat{\boldsymbol{\mu}}$ be the sample average estimate of $\boldsymbol{\mu}$, and $\hat{\boldsymbol{\Sigma}}$ the sample covariance estimate of $\boldsymbol{\Sigma}$. In the remainder, we will assume that the estimate $\hat{\boldsymbol{\Sigma}}$ is reasonably accurate such that there is no uncertainty about it. This assumption is justified since the estimation error in $\hat{\boldsymbol{\mu}}$ by far outweighs the estimation error in $\hat{\boldsymbol{\Sigma}}$, see e.g. [16]. Thus, we may view the uncertainty set for the covariance matrix as a singleton, $\Theta_{\boldsymbol{\Sigma}}=\{\hat{\boldsymbol{\Sigma}}\}$. We note that all the following results can be generalized to cases in which $\Theta_{\boldsymbol{\Sigma}}$ is not a singleton. This, however, leads to more convoluted model formulations. If the stock returns are serially independent and identically distributed, we can invoke the Central Limit Theorem to conclude that the sample mean $\hat{\boldsymbol{\mu}}$ is approximately normally distributed. Henceforth we will thus assume that

$$
\hat{\boldsymbol{\mu}} \sim \mathcal{N}(\boldsymbol{\mu}, \boldsymbol{\Lambda}), \quad \boldsymbol{\Lambda}=(1 / E) \boldsymbol{\Sigma},
$$

where $E$ is the number of historical samples used to calculate $\hat{\boldsymbol{\mu}}$. It is therefore natural to assume an ellipsoidal uncertainty set for the means,

$$
\Theta_{\boldsymbol{\mu}}=\left\{\boldsymbol{\mu}:(\boldsymbol{\mu}-\hat{\boldsymbol{\mu}})^{T} \boldsymbol{\Lambda}^{-1}(\boldsymbol{\mu}-\hat{\boldsymbol{\mu}}) \leq \kappa^{2}\right\},
$$

where $\kappa=\sqrt{q /(1-q)}$ for some $q \in[0,1)$. The confidence level $q$ has an analog interpretation as the parameter $p$ in (6). Using the above specifications of the uncertainty sets, problem (8) reduces to

$$
\max _{\boldsymbol{w} \in \mathbb{R}^{n}}\left\{\boldsymbol{w}^{T} \hat{\boldsymbol{\mu}}-\kappa\left\|\boldsymbol{\Lambda}^{1 / 2} \boldsymbol{w}\right\|_{2}-\delta\left\|\hat{\boldsymbol{\Sigma}}^{1 / 2} \boldsymbol{w}\right\|_{2} \mid \boldsymbol{w}^{T} \mathbf{1}=1, \boldsymbol{l} \leq \boldsymbol{w} \leq \boldsymbol{u}\right\},
$$

see [13]. By using the relations (9), one easily verifies that (11) is equivalent to

$$
\max _{\boldsymbol{w} \in \mathbb{R}^{n}}\left\{\boldsymbol{w}^{T} \hat{\boldsymbol{\mu}}-\left(\frac{\kappa}{\sqrt{E}}+\delta\right)\left\|\hat{\boldsymbol{\Sigma}}^{1 / 2} \boldsymbol{w}\right\|_{2} \mid \boldsymbol{w}^{T} \mathbf{1}=1, \boldsymbol{l} \leq \boldsymbol{w} \leq \boldsymbol{u}\right\} .
$$

This problem is equivalent to (7) with the risk parameter $\delta$ shifted by $\kappa / \sqrt{E}$. Therefore, it is also equivalent to the standard Markowitz model. Hence, seemingly nothing has been gained by incorporating parameter uncertainty into the model (7). 
Ceria and Stubbs [13] demonstrate that robust optimization can nevertheless be used to systematically improve on the common Markowitz portfolios (which are optimal in (2), (7), and (11)). The key idea is to replace the elliptical uncertainty set (10) by a less conservative one. Since the estimated expected returns $\hat{\boldsymbol{\mu}}$ are symmetrically distributed around $\boldsymbol{\mu}$, we expect that the estimation errors cancel out when summed over all stocks. It may be more natural and less pessimistic to explicitly incorporate this expectation into the uncertainty model. To this end, Ceria and Stubbs set

$$
\Theta_{\boldsymbol{\mu}}=\left\{\boldsymbol{\mu}:(\boldsymbol{\mu}-\hat{\boldsymbol{\mu}})^{T} \boldsymbol{\Lambda}^{-1}(\boldsymbol{\mu}-\hat{\boldsymbol{\mu}}) \leq \kappa^{2}, \mathbf{1}^{T}(\boldsymbol{\mu}-\hat{\boldsymbol{\mu}})=0\right\} .
$$

With this new uncertainty set problem (8) reduces to

$$
\max _{\boldsymbol{w} \in \mathbb{R}^{n}}\left\{\boldsymbol{w}^{T} \hat{\boldsymbol{\mu}}-\kappa\left\|\boldsymbol{\Omega}^{1 / 2} \boldsymbol{w}\right\|_{2}-\delta\left\|\hat{\boldsymbol{\Sigma}}^{1 / 2} \boldsymbol{w}\right\|_{2} \mid \boldsymbol{w}^{T} \mathbf{1}=1, \boldsymbol{l} \leq \boldsymbol{w} \leq \boldsymbol{u}\right\},
$$

where

$$
\boldsymbol{\Omega}=\boldsymbol{\Lambda}-\frac{1}{\mathbf{1}^{T} \boldsymbol{\Lambda} \mathbf{1}} \boldsymbol{\Lambda} \mathbf{1 1 ^ { T }} \boldsymbol{\Lambda},
$$

see [13]. A formal derivation of the optimization problem (13) is provided in Theorem A.1 in Appendix A.

\subsection{Uncertainty Sets with Support Information}

For ease of exposition, consider again the basic model of Section 2.1. When the uncertainty set $\Theta_{\boldsymbol{r}}$ becomes excessively large, as is the case when $\delta \rightarrow+\infty$ or, equivalently, when $p \rightarrow 1$ (see (5)), $\Theta_{\boldsymbol{r}}$ may extend beyond the support of $\tilde{\boldsymbol{r}}$, which coincides with the positive orthant of $\mathbb{R}^{n}$. The resulting portfolios can then become unnecessarily conservative. To overcome this deficiency, we modify $\Theta_{r}$ defined in (4) by including a non-negativity constraint

$$
\Theta_{\boldsymbol{r}}^{+}=\left\{\boldsymbol{r} \geq \mathbf{0}:(\boldsymbol{r}-\boldsymbol{\mu})^{T} \boldsymbol{\Sigma}^{-1}(\boldsymbol{r}-\boldsymbol{\mu}) \leq \delta^{2}\right\}
$$

It can be shown that problem (3) with $\Theta_{r}$ replaced by $\Theta_{r}^{+}$is equivalent to

$$
\max _{\boldsymbol{w}, \boldsymbol{s} \in \mathbb{R}^{n}}\left\{\boldsymbol{\mu}^{T}(\boldsymbol{w}-\boldsymbol{s})-\delta\left\|\boldsymbol{\Sigma}^{1 / 2}(\boldsymbol{w}-\boldsymbol{s})\right\|_{2} \mid \boldsymbol{w}^{T} \mathbf{1}=1, \boldsymbol{s} \geq \mathbf{0}, \boldsymbol{l} \leq \boldsymbol{w} \leq \boldsymbol{u}\right\} .
$$

Remark 2.1 (Relation to coherent risk measures) Problem (15) can be shown to implicitly minimize a coherent downside risk measure [3] associated with the underlying uncertainty set. Natarajan et al. [37] show that there exists a one-to-one correspondence between uncertainty sets and risk measures (see also [87). In what follows, we will briefly explain this correspondence in the context of problem (15). Introduce a linear space of random variables

$$
\mathcal{V}=\left\{\boldsymbol{w}^{T} \tilde{\boldsymbol{r}}: \boldsymbol{w} \in \mathbb{R}^{n}\right\},
$$

and define the risk measure $\rho: \mathcal{V} \rightarrow \mathbb{R}$ through

$$
\begin{aligned}
\rho\left(\boldsymbol{w}^{T} \tilde{\boldsymbol{r}}\right) & =\max _{\boldsymbol{r}}\left\{-\boldsymbol{w}^{T} \boldsymbol{r} \mid \boldsymbol{r} \in \Theta_{\boldsymbol{r}}^{+}\right\} \\
& =\min _{\boldsymbol{s} \geq \mathbf{0}}-\boldsymbol{\mu}^{T}(\boldsymbol{w}-\boldsymbol{s})+\delta\left\|\boldsymbol{\Sigma}^{1 / 2}(\boldsymbol{w}-\boldsymbol{s})\right\|_{2} .
\end{aligned}
$$

It can be seen that problem (15) is equivalent to the risk minimization problem

$$
\min _{\boldsymbol{w}}\left\{\rho\left(\boldsymbol{w}^{T} \tilde{\boldsymbol{r}}\right) \mid \mathbf{1}^{T} \boldsymbol{w}=1, \boldsymbol{l} \leq \boldsymbol{w} \leq \boldsymbol{u}\right\}
$$


Since the feasible set in (17) is a subset of the support of $\tilde{\boldsymbol{r}}$, the risk measure $\rho$ is coherent, see [37, Theorem 4]. Moreover, $\rho$ can be viewed as a downside risk measure since it evaluates to worst-case return over an uncertainty set centered around the expected asset return vector.

As in Section 2.2, model (15) may be improved by immunizing it against the uncertainty in the distributional input parameters. Using similar arguments as in Theorem A.1, it can be shown that the parameter robust variant of problem (15),

$$
\max _{\boldsymbol{w}, \boldsymbol{s}}\left\{\min _{\boldsymbol{\mu} \in \Theta_{\boldsymbol{\mu}}} \boldsymbol{\mu}^{T}(\boldsymbol{w}-\boldsymbol{s})-\delta \max _{\boldsymbol{\Sigma} \in \Theta_{\boldsymbol{\Sigma}}}\left\|\boldsymbol{\Sigma}^{1 / 2}(\boldsymbol{w}-\boldsymbol{s})\right\|_{2} \mid \boldsymbol{w}^{T} \mathbf{1}=1, \boldsymbol{s} \geq \mathbf{0}, \boldsymbol{l} \leq \boldsymbol{w} \leq \boldsymbol{u}\right\},
$$

is equivalent to

$$
\max _{\boldsymbol{w}, \boldsymbol{s}, \boldsymbol{v}}\left\{\hat{\boldsymbol{\mu}}^{T} \boldsymbol{v}-\kappa\left\|\boldsymbol{\Omega}^{1 / 2} \boldsymbol{v}\right\|_{2}-\delta\left\|\hat{\boldsymbol{\Sigma}}^{1 / 2} \boldsymbol{v}\right\|_{2} \mid \boldsymbol{w}^{T} \mathbf{1}=1, \boldsymbol{w}-\boldsymbol{s}=\boldsymbol{v}, \boldsymbol{s} \geq \mathbf{0}, \boldsymbol{l} \leq \boldsymbol{w} \leq \boldsymbol{u}\right\} .
$$

We note that we could have directly obtained (19) from the basic model (3) by defining the uncertainty set for the returns as

$$
\Theta_{\boldsymbol{r}, \boldsymbol{\mu}}^{+}=\left\{\boldsymbol{r} \geq \mathbf{0}: \exists \boldsymbol{\mu} \in \Theta_{\boldsymbol{\mu}},(\boldsymbol{r}-\boldsymbol{\mu})^{T} \boldsymbol{\Sigma}^{-1}(\boldsymbol{r}-\boldsymbol{\mu}) \leq \delta^{2}\right\}
$$

where $\Theta_{\boldsymbol{\mu}}$ is defined as in (12). The uncertainty set $\Theta_{\boldsymbol{r}, \boldsymbol{\mu}}^{+}$accounts for the uncertainty in the returns whilst taking into consideration that the centroid $\boldsymbol{\mu}$ of $\Theta_{\boldsymbol{r}}^{+}$, as defined in (14), has to be estimated and is therefore also subject to uncertainty.

Problem (19) implicitly minimizes a coherent risk measure associated with the uncertainty set $\Theta_{\boldsymbol{r}, \boldsymbol{\mu}}^{+}$. Coherency holds since $\Theta_{\boldsymbol{r}, \boldsymbol{\mu}}^{+}$is a subset of the support of $\tilde{\boldsymbol{r}}$, see Remark 2.1. Some risk-tolerant investors may not want to minimize a risk measure without imposing a constraint on the portfolio return. Taking into account the uncertainty in the expected asset returns motivates us to constrain the worst-case expected portfolio return,

$$
\min _{\boldsymbol{\mu} \in \Theta_{\mu}} \boldsymbol{w}^{T} \boldsymbol{\mu} \geq \mu_{\text {target }},
$$

where $\mu_{\text {target }}$ represents the return target the investor wishes to attain in average. This semi-infinite constraint can be reformulated as a second-order cone constraint of the form

$$
\boldsymbol{w}^{T} \hat{\boldsymbol{\mu}}-\kappa\left\|\boldsymbol{\Omega}^{1 / 2} \boldsymbol{w}\right\|_{2} \geq \mu_{\text {target }} .
$$

The optimal portfolios obtained from problem (19), with or without the return target constraint (21), provide certain performance guarantees. They exhibit a non-inferiority property in the sense that, as long as the asset returns materialize within the prescribed uncertainty set, the realized portfolio return never falls below the optimal value of problem (19). However, no guarantees are given when the asset returns are realized outside of the uncertainty set.

In Section 3 we suggest the use of derivatives to enforce strong performance guarantees, which will complement the weak guarantees provided by the non-inferiority property.

\section{Insured Robust Portfolio Optimization}

Since their introduction in the second half of the last century, options have been praised for their ability to give stock holders protection against adverse market fluctuations [31]. 
A standard option contract is determined by the following parameters: the premium or price of the option, the underlying security, the expiration date, and the strike price. A put (call) option gives the option holder the right, but not the obligation, to sell to (buy from) the option writer the underlying security by the expiration date and at the prescribed strike price. American options can be exercised at any time up to the expiration date, whereas European options can be exercised only on the expiration date itself. We will only work with European options, which expire at the end of investment horizon, that is, at time $T$. We restrict attention to these instruments because of their simplicity and since they fit naturally in the single period portfolio optimization framework of the previous section.

We now briefly illustrate how options can be used to insure a stock portfolio. An option's payoff function represents its value at maturity as a function of the underlying stock price $S_{T}$. For put and call options with strike price $K$, the payoff functions are thus given by

$$
V_{\text {put }}\left(S_{T}\right)=\max \left\{0, K-S_{T}\right\} \quad \text { and } \quad V_{\text {call }}\left(S_{T}\right)=\max \left\{0, S_{T}-K\right\},
$$

respectively. Assume now that we hold a portfolio of a single long stock and a put option on this stock with strike price $K$. Then, the payoff of the portfolio amounts to

$$
V_{\mathrm{pf}}\left(S_{T}\right)=S_{T}+V_{\mathrm{put}}\left(S_{T}\right)=\max \left\{S_{T}, K\right\} .
$$

This shows that the put option with strike price $K$ prevents the portfolio value at maturity from dropping below $K$. Of course, this insurance comes at the cost of the option premium, which has to be paid at the time when the option contract is negotiated.

Similarly, assume that we hold a portfolio of a single shorted stock and a call option on this stock with strike price $K$. Then, the payoff function of this portfolio is

$$
V_{\mathrm{pf}}\left(S_{T}\right)=-S_{T}+V_{\text {call }}\left(S_{T}\right)=\max \left\{-S_{T},-K\right\}
$$

which insures the portfolio value at maturity against falling below $-K$.

Although we focus on European options expiring at time $T$, all models to be developed in this paper remain valid for American options exercisable at time $T$. We emphasize that the timing flexibility of American options cannot be exploited in the single-period setting under consideration, and therefore American options are usually too expensive for our purposes. Nevertheless, if there are only very few European options expiring at the end of the investment horizon, it may be beneficial to include American options into our portfolio to increase the spectrum of available strike prices.

\subsection{Robust Portfolio Optimization with Options}

Assume that there are $m$ European options in our market, each of which has one of the $n$ stocks as an underlying security. We denote the initial investment in the options by the vector $\boldsymbol{w}^{\boldsymbol{d}} \in \mathbb{R}^{m}$. The component $w_{i}^{d}$ denotes the percentage of total wealth which is invested in the $i$ th option at time $t=0$. A portfolio is now completely characterized by a joint vector $\left(\boldsymbol{w}, \boldsymbol{w}^{\boldsymbol{d}}\right) \in \mathbb{R}^{n+m}$, whose elements add up to 1 . In what follows, we will forbid short-sales of options and therefore require that $\boldsymbol{w}^{\boldsymbol{d}} \geq \mathbf{0}$. Short-selling of options can be very risky, and therefore the imposed restriction should be in line with the preferences of a risk-averse investor.

The return $\tilde{r}_{p}$ of some portfolio $\left(\boldsymbol{w}, \boldsymbol{w}^{\boldsymbol{d}}\right)$ is given by

$$
\tilde{r}_{p}=\boldsymbol{w}^{T} \tilde{\boldsymbol{r}}+\left(\boldsymbol{w}^{\boldsymbol{d}}\right)^{T} \tilde{\boldsymbol{r}}^{\boldsymbol{d}},
$$


where $\tilde{\boldsymbol{r}}^{\boldsymbol{d}}$ represents the vector of option returns. It is important to note that $\tilde{\boldsymbol{r}}^{\boldsymbol{d}}$ is uniquely determined by $\tilde{\boldsymbol{r}}$, that is, there exists a function $f: \mathbb{R}^{n} \rightarrow \mathbb{R}^{m}$ such that $\tilde{\boldsymbol{r}}^{\boldsymbol{d}} \equiv f(\tilde{\boldsymbol{r}})$.

Let option $j$ be a call with strike price $K_{j}$ on the underlying stock $i$, and denote the return and the initial price of the option by $\tilde{r}_{j}^{d}$ and $C_{j}$, respectively. If $S_{0}^{i}$ denotes the initial price of stock $i$, then its end-of-period price can be expressed as $S_{0}^{i} \tilde{r}_{i}$. Using the above notation, we can now explicitly express the return $\tilde{r}_{j}^{d}$ as a convex piece-wise linear function of $\tilde{r}_{i}$,

$$
\begin{aligned}
f_{j}(\tilde{\boldsymbol{r}}) & =\frac{1}{C_{j}} \max \left\{0, S_{0}^{i} \tilde{r}_{i}-K_{j}\right\} \\
& =\max \left\{0, a_{j}+b_{j} \tilde{r}_{i}\right\}, \text { with } a_{j}=-\frac{K_{j}}{C_{j}}<0 \text { and } b_{j}=\frac{S_{0}^{i}}{C_{j}}>0 .
\end{aligned}
$$

Similarly, if $\tilde{r}_{j}^{d}$ is the return of a put option with price $P_{j}$ and strike price $K_{j}$ on the underlying stock $i$, then $\tilde{r}_{j}^{d}$ is representable as a slightly different convex piece-wise linear function of $\tilde{r}_{i}$,

$$
f_{j}(\tilde{\boldsymbol{r}})=\max \left\{0, a_{j}+b_{j} \tilde{r}_{i}\right\}, \text { with } a_{j}=\frac{K_{j}}{P_{j}}>0 \text { and } b_{j}=-\frac{S_{0}^{i}}{P_{j}}<0 \text {. }
$$

Using the above notation, we can write the vector of option returns $\tilde{\boldsymbol{r}}^{d}$ compactly as

$$
\tilde{\boldsymbol{r}}^{\boldsymbol{d}}=f(\tilde{\boldsymbol{r}})=\max \{\mathbf{0}, \boldsymbol{a}+\mathbf{B} \tilde{\boldsymbol{r}}\},
$$

where $\boldsymbol{a} \in \mathbb{R}^{m}, \mathbf{B} \in \mathbb{R}^{m \times n}$ are known constants determined through (24a) and (24b), and 'max' denotes the component-wise maximization operator.

As in Section 2.3, we adopt the view that the investor wishes to maximize the worstcase portfolio return whilst assuming that the stock returns $\tilde{\boldsymbol{r}}$ will materialize within the uncertainty set $\Theta_{r}^{+}$as defined in (14). This problem can be formalized as

$$
\max _{\boldsymbol{w}, \boldsymbol{w}^{\boldsymbol{d}}}\left\{\min _{\substack{\boldsymbol{r} \in \Theta_{r} \\ \boldsymbol{r}^{d}=f(\boldsymbol{r})}} \boldsymbol{w}^{T} \boldsymbol{r}+\left(\boldsymbol{w}^{\boldsymbol{d}}\right)^{T} \boldsymbol{r}^{\boldsymbol{d}} \mid \mathbf{1}^{T} \boldsymbol{w}+\mathbf{1}^{T} \boldsymbol{w}^{\boldsymbol{d}}=1, \boldsymbol{l} \leq \boldsymbol{w} \leq \boldsymbol{u}, \boldsymbol{w}^{\boldsymbol{d}} \geq \mathbf{0}\right\},
$$

which is equivalent to

$$
\begin{array}{ll}
\underset{\boldsymbol{w}, \boldsymbol{w}^{\boldsymbol{d}}, \phi}{\operatorname{maximize}} & \phi \\
\text { subject to } & \boldsymbol{w}^{T} \boldsymbol{r}+\left(\boldsymbol{w}^{\boldsymbol{d}}\right)^{T} \boldsymbol{r}^{\boldsymbol{d}} \geq \phi \\
& \mathbf{1}^{T} \boldsymbol{w}+\mathbf{1}^{T} \boldsymbol{w}^{\boldsymbol{d}}=1
\end{array} \quad \forall \boldsymbol{r} \in \Theta_{\boldsymbol{r}}^{+}, \boldsymbol{r}^{\boldsymbol{d}}=f(\boldsymbol{r})
$$

Note that the worst-case objective is reexpressed in terms of the semi-infinite constraint $(27 \mathrm{~b})$, and at optimality, $\phi$ represents the worst-case portfolio return. In the remainder we will work with the epigraph formulation (27) instead of the max-min formulation (26) because it enables us to incorporate portfolio insurance constraints in a convenient way, see Section 3.2.

The constraint (27b) looks intractable, but it can be reformulated in terms of finitely many conic constraints. 
Theorem 3.1 Problem (27) is equivalent to

$$
\begin{array}{ll}
\underset{\boldsymbol{w}, \boldsymbol{w}^{\boldsymbol{d}}, \boldsymbol{y}, \boldsymbol{s}, \phi}{\operatorname{maximize}} & \phi \\
\text { subject to } & \boldsymbol{\mu}^{T}\left(\boldsymbol{w}+\mathbf{B}^{T} \boldsymbol{y}-\boldsymbol{s}\right)-\delta\left\|\boldsymbol{\Sigma}^{1 / 2}\left(\boldsymbol{w}+\mathbf{B}^{T} \boldsymbol{y}-\boldsymbol{s}\right)\right\|_{2}+\boldsymbol{a}^{T} \boldsymbol{y} \geq \phi \\
& \mathbf{1}^{T} \boldsymbol{w}+\mathbf{1}^{T} \boldsymbol{w}^{\boldsymbol{d}}=1 \\
& \mathbf{0} \leq \boldsymbol{y} \leq \boldsymbol{w}^{\boldsymbol{d}}, \boldsymbol{s} \geq \mathbf{0} \\
& \boldsymbol{l} \leq \boldsymbol{w} \leq \boldsymbol{u}, \boldsymbol{w}^{\boldsymbol{d}} \geq \mathbf{0},
\end{array}
$$

which is a tractable second-order cone program.

Proof Assume first that $\delta>0$. We observe that the semi-infinite constraint (27b) can be reexpressed in terms of the solution of a subordinate minimization problem,

$$
\min _{\substack{\boldsymbol{r} \in \Theta_{r} \\ \boldsymbol{r}^{d}=f(\boldsymbol{r})}} \boldsymbol{w}^{T} \boldsymbol{r}+\left(\boldsymbol{w}^{\boldsymbol{d}}\right)^{T} \boldsymbol{r}^{\boldsymbol{d}} \geq \phi .
$$

By using the definitions of the function $f$ and the set $\Theta_{\boldsymbol{r}}^{+}$, we obtain a more explicit representation for this subordinate problem.

$$
\begin{array}{ll}
\min _{\boldsymbol{r}, \boldsymbol{r}^{\boldsymbol{d}}} & \boldsymbol{w}^{T} \boldsymbol{r}+\left(\boldsymbol{w}^{\boldsymbol{d}}\right)^{T} \boldsymbol{r}^{\boldsymbol{d}} \\
\text { subject to } & \left\|\boldsymbol{\Sigma}^{-1 / 2}(\boldsymbol{r}-\boldsymbol{\mu})\right\|_{2} \leq \delta \\
& \boldsymbol{r} \geq \mathbf{0} \\
& \boldsymbol{r}^{\boldsymbol{d}} \geq \mathbf{0} \\
& \boldsymbol{r}^{\boldsymbol{d}} \geq \boldsymbol{a}+\mathbf{B} \boldsymbol{r}
\end{array}
$$

For any fixed portfolio vector $\left(\boldsymbol{w}, \boldsymbol{w}^{\boldsymbol{d}}\right)$ feasible in (27), problem (30) represents a convex second-order cone program. Note that since $\boldsymbol{w}^{\boldsymbol{d}} \geq \mathbf{0}$ for any admissible portfolio, (30) has an optimal solution $\left(\boldsymbol{r}, \boldsymbol{r}^{\boldsymbol{d}}\right)$ which satisfies the relation (25). The dual problem associated with (30) reads:

$$
\begin{array}{cl}
\max _{\boldsymbol{y} \in \mathbb{R}^{m}, \boldsymbol{s} \in \mathbb{R}^{n}} & \boldsymbol{\mu}^{T}\left(\boldsymbol{w}+\mathbf{B}^{T} \boldsymbol{y}-\boldsymbol{s}\right)-\delta\left\|\boldsymbol{\Sigma}^{1 / 2}\left(\boldsymbol{w}+\mathbf{B}^{T} \boldsymbol{y}-\boldsymbol{s}\right)\right\|_{2}+\boldsymbol{a}^{T} \boldsymbol{y} \\
\text { subject to } & \mathbf{0} \leq \boldsymbol{y} \leq \boldsymbol{w}^{\boldsymbol{d}}, \boldsymbol{s} \geq \mathbf{0}
\end{array}
$$

Note that strong conic duality holds since the primal problem (30) is strictly feasible for $\delta>0$, see [2, 27]. Thus, both the primal and dual problems (30) and (31) are feasible and share the same objective values at optimality. This allows us to replace the inner minimization problem in (29) by the maximization problem (31). The requirement that the optimal value of (31) be larger than or equal to $\phi$ is equivalent to the assertion that there exist $\boldsymbol{y} \in \mathbb{R}^{m}, \boldsymbol{s} \in \mathbb{R}^{n}$ feasible in (31) whose objective value is larger than or equal to $\phi$. This justifies the constraints (28b) and (28d). All other constraints and the objective function in (28) are the same as in (27), and thus the two problems are equivalent.

We now assume that $\delta=0$. Then, by definition, the uncertainty set $\Theta_{\boldsymbol{r}}^{+}=\{\boldsymbol{\mu}\}$ and 
$\boldsymbol{r}^{\boldsymbol{d}}=f(\boldsymbol{\mu})$. Therefore, constraint $(27 \mathrm{~b})$ reduces to

$$
\begin{aligned}
& \boldsymbol{\mu}^{T} \boldsymbol{w}+f(\boldsymbol{\mu})^{T} \boldsymbol{w}^{\boldsymbol{d}} \geq \phi \\
\Longleftrightarrow & \boldsymbol{\mu}^{T} \boldsymbol{w}+(\max \{\boldsymbol{0}, \boldsymbol{a}+\mathbf{B} \boldsymbol{\mu}\})^{T} \boldsymbol{w}^{\boldsymbol{d}} \geq \phi \\
\Longleftrightarrow & \boldsymbol{\mu}^{T} \boldsymbol{w}+\max _{\mathbf{0} \leq \boldsymbol{y} \leq \boldsymbol{w}^{\boldsymbol{d}}}\left\{\boldsymbol{a}^{T} \boldsymbol{y}+\boldsymbol{\mu}^{T} \mathbf{B}^{T} \boldsymbol{y}\right\} \geq \phi \\
\Longleftrightarrow & \max _{\substack{\boldsymbol{0} \leq \boldsymbol{y} \leq \boldsymbol{w}^{\boldsymbol{d}} \\
\boldsymbol{s} \geq \boldsymbol{0}}}\left\{\boldsymbol{\mu}^{T}\left(\boldsymbol{w}+\mathbf{B}^{T} \boldsymbol{y}-\boldsymbol{s}\right)+\boldsymbol{a}^{T} \boldsymbol{y}\right\} \geq \phi,
\end{aligned}
$$

where the last equivalence holds because $\boldsymbol{\mu} \geq \mathbf{0}$. Constraint (27b) is thus equivalent to $(28 \mathrm{~b})$ and $(28 \mathrm{~d})$.

Observe that in the absence of options we must set $\boldsymbol{w}^{d}=\mathbf{0}$, which implies via constraint (28d) that $\boldsymbol{y}=\mathbf{0}$. Thus, (28) reduces to (15), that is, the robust portfolio optimization problem of a stock only portfolio.

We note that Lutgens et al. [30] propose a robust portfolio optimization model that incorporates options and also allows short-sales of options. However, their problem reformulation contains, in the worst case, an exponential amount of second-order constraints whereas our reformulation (28) only contains a single conic constraint at the cost of excluding short-sales of options.

As in Section 2.3, one can immunize model (27) against estimation errors in $\hat{\boldsymbol{\mu}}$. If we replace the uncertainty set $\Theta_{\boldsymbol{r}}^{+}$by $\Theta_{\boldsymbol{r}, \boldsymbol{\mu}}^{+}$defined in (20), then problem (27) reduces to the following second-order cone program similar to (28).

$$
\begin{aligned}
\operatorname{maximize} & \phi \\
\text { subject to } & \hat{\boldsymbol{\mu}}^{T} \boldsymbol{v}-\kappa\left\|\boldsymbol{\Omega}^{1 / 2} \boldsymbol{v}\right\|_{2}-\delta\left\|\hat{\boldsymbol{\Sigma}}^{1 / 2} \boldsymbol{v}\right\|_{2}+\boldsymbol{a}^{T} \boldsymbol{y} \geq \phi \\
& \boldsymbol{w}+\mathbf{B}^{T} \boldsymbol{y}-\boldsymbol{s}=\boldsymbol{v}, \text { and }(28 \mathrm{c}),(28 \mathrm{~d}),(28 \mathrm{e})
\end{aligned}
$$

This model guarantees the optimal portfolio return to exceed $\phi$ conditional on the stock returns $\tilde{\boldsymbol{r}}$ being realized within the uncertainty set $\Theta_{\boldsymbol{r}, \boldsymbol{\mu}}^{+}$. In what follows, we will thus refer to $\phi$ as the conditional worst-case return.

\subsection{Robust Portfolio Optimization with Insurance Guarantees}

We now augment model (32) by requiring the realized portfolio return to exceed some fraction $\theta \in[0,1]$ of $\phi$ under every possible realization of the return vector $\tilde{\boldsymbol{r}}$. This requirement is enforced through a semi-infinite constraint of the form

$$
\boldsymbol{w}^{T} \boldsymbol{r}+\left(\boldsymbol{w}^{\boldsymbol{d}}\right)^{T} \boldsymbol{r}^{\boldsymbol{d}} \geq \theta \phi \quad \forall \boldsymbol{r} \geq \mathbf{0}, \boldsymbol{r}^{\boldsymbol{d}}=f(\boldsymbol{r}) .
$$

Model (32) with the extra constraint (33) provides two layers of guarantees: the weak non-inferiority guarantee applies as long as the returns are realized within the uncertainty set, while the strong portfolio insurance guarantee (33) also covers cases in which the stock returns are realized outside of $\Theta_{\boldsymbol{r}, \boldsymbol{\mu}}^{+} \cdot{ }^{1}$ The level of the portfolio insurance guarantee is expressed as a percentage $\theta$ of the conditional worst-case portfolio return $\phi$, which can be interpreted as the level of the non-inferiority guarantee. This reflects the idea that the

\footnotetext{
${ }^{1}$ In reality one has to also consider counterparty risk of the options, but this is beyond the scope of this paper.
} 
derivative insurance strategy only has to hedge against certain extreme scenarios, which are not already covered by the non-inferiority guarantee. It also prevents the portfolio insurance from being overly expensive. The Insured Robust Portfolio Optimization model can be formulated as

$$
\begin{array}{cll}
\underset{\boldsymbol{w}, \boldsymbol{w}^{\boldsymbol{d}}, \phi}{\operatorname{maximize}} & \phi \\
\text { subject to } & \boldsymbol{w}^{T} \boldsymbol{r}+\left(\boldsymbol{w}^{\boldsymbol{d}}\right)^{T} \boldsymbol{r}^{\boldsymbol{d}} \geq \phi & \forall \boldsymbol{r} \in \Theta_{\boldsymbol{r}, \boldsymbol{\mu}}^{+}, \boldsymbol{r}^{\boldsymbol{d}}=f(\boldsymbol{r}) \\
& \boldsymbol{w}^{T} \boldsymbol{r}+\left(\boldsymbol{w}^{\boldsymbol{d}}\right)^{T} \boldsymbol{r}^{\boldsymbol{d}} \geq \theta \phi & \forall \boldsymbol{r} \geq \mathbf{0}, \boldsymbol{r}^{\boldsymbol{d}}=f(\boldsymbol{r}) \\
& \mathbf{1}^{T} \boldsymbol{w}+\mathbf{1}^{T} \boldsymbol{w}^{\boldsymbol{d}}=1 & \\
& \boldsymbol{l} \leq \boldsymbol{w} \leq \boldsymbol{u}, \boldsymbol{w}^{\boldsymbol{d}} \geq \mathbf{0} . &
\end{array}
$$

Note that the conditional worst-case return $\phi$ drops when the uncertainty set $\Theta_{\boldsymbol{r}, \boldsymbol{\mu}}^{+}$ increases. At the same time, the required insurance level decreases, and hence the insurance premium drops as well. This manifests the tradeoff between the non-inferiority and insurance guarantees. In Proposition 3.1 below we show that when the highest possible uncertainty is assigned to the returns (by setting $p=1$, see (5)), or the highest insurance guarantee is demanded (by setting $\theta=1$ ), the same optimal conditional worst-case return is obtained. Intuitively, this can be explained as follows. When the uncertainty set covers the whole support, then the insurance guarantee adds nothing to the non-inferiority guarantee. Conversely, the highest possible insurance is independent of the size of the uncertainty set.

Proposition 3.1 If $\boldsymbol{u} \geq \mathbf{0}$, then the optimal objective value of problem (34) for $p=1$ coincides with the optimal value obtained for $\theta=1$.

Proof Since $\boldsymbol{u} \geq \mathbf{0}$, there are feasible portfolios with $\boldsymbol{w} \geq \mathbf{0}$. Thus, $\phi \geq \theta \phi \geq 0$ at optimality. For $p=1$, the uncertainty sets in (34b) and (34c) coincide, which implies that $(34 \mathrm{c})$ becomes redundant. For $\theta=1$, on the other hand, (34b) becomes redundant. In both cases we end up with the same constraint set. Thus, the claim follows.

Although we exclusively use uncertainty sets of the type (20), the models in this paper do not rely on any assumptions about the size or shape of $\Theta_{\boldsymbol{r}, \boldsymbol{\mu}}^{+}$and can be extended to almost any other geometry. We note that for the models to be tractable, it must be possible to describe $\Theta_{\boldsymbol{r}, \boldsymbol{\mu}}^{+}$through finitely many linear or conic constraints.

Problem (34) involves two semi-infinite constraints: (34b) and (34c). In Theorem 3.2 we show that (34) still has a reformulation as a tractable conic optimization problem.

Theorem 3.2 Problem (34) is equivalent to the following second-order cone program.

$$
\begin{array}{ll}
\text { maximize } & \phi \\
\text { subject to } & \hat{\boldsymbol{\mu}}^{T} \boldsymbol{v}-\kappa\left\|\boldsymbol{\Omega}^{1 / 2} \boldsymbol{v}\right\|_{2}-\delta\left\|\hat{\boldsymbol{\Sigma}}^{1 / 2} \boldsymbol{v}\right\|_{2}+\boldsymbol{a}^{T} \boldsymbol{y} \geq \phi \\
& \boldsymbol{a}^{T} \boldsymbol{z} \geq \theta \phi \\
& \boldsymbol{w}+\mathbf{B}^{T} \boldsymbol{y}-\boldsymbol{s}=\boldsymbol{v} \\
& \boldsymbol{w}+\mathbf{B}^{T} \boldsymbol{z} \geq \mathbf{0} \\
& \mathbf{1}^{T} \boldsymbol{w}+\mathbf{1}^{T} \boldsymbol{w}^{\boldsymbol{d}}=1 \\
& \mathbf{0} \leq \boldsymbol{y} \leq \boldsymbol{w}^{\boldsymbol{d}}, \mathbf{0} \leq \boldsymbol{z} \leq \boldsymbol{w}^{\boldsymbol{d}}, \\
& \boldsymbol{s} \geq \mathbf{0}, \boldsymbol{w}^{\boldsymbol{d}} \geq \mathbf{0}, \boldsymbol{l} \leq \boldsymbol{w} \leq \boldsymbol{u} .
\end{array}
$$


Proof We already know how to reexpress (34b) in terms of finitely many conic constraints. Therefore, we now focus on the reformulation of (34c).

As usual, we first reformulate (34c) in terms of a subordinate minimization problem,

$$
\min _{\substack{r \geq 0 \\ r^{d}=f(r)}} \boldsymbol{w}^{T} \boldsymbol{r}+\left(\boldsymbol{w}^{\boldsymbol{d}}\right)^{T} \boldsymbol{r}^{\boldsymbol{d}} \geq \theta \phi .
$$

By using the definition of the function $f$ and the fact that $\boldsymbol{w}^{\boldsymbol{d}} \geq \mathbf{0}$, the left-hand side of (36) can be reexpressed as the linear program

$$
\begin{array}{ll}
\min _{\boldsymbol{r}, \boldsymbol{r}^{d}} & \boldsymbol{w}^{T} \boldsymbol{r}+\left(\boldsymbol{w}^{\boldsymbol{d}}\right)^{T} \boldsymbol{r}^{\boldsymbol{d}} \\
\text { subject to } & \boldsymbol{r} \geq \mathbf{0} \\
& \boldsymbol{r}^{\boldsymbol{d}} \geq \mathbf{0} \\
& \boldsymbol{r}^{\boldsymbol{d}} \geq \boldsymbol{a}+\mathbf{B} \boldsymbol{r} .
\end{array}
$$

The dual of problem (37) reads

$$
\begin{array}{ll}
\max _{\boldsymbol{z} \in \mathbb{R}^{m}} & \boldsymbol{a}^{T} \boldsymbol{z} \\
\text { subject to } & \boldsymbol{w}+\mathbf{B}^{T} \boldsymbol{z} \geq \mathbf{0} \\
& \mathbf{0} \leq \boldsymbol{z} \leq \boldsymbol{w}^{\boldsymbol{d}} .
\end{array}
$$

Strong linear duality holds because the primal problem (37) is manifestly feasible. Therefore, the optimal objective value of problem (38) coincides with that of problem (37), and we can substitute (38) into the constraint (36). This leads to the postulated reformulation in (35).

Note that problem (35) implicitly minimizes a coherent risk measure determined through the uncertainty set

$$
\left\{\left(\boldsymbol{r}, \boldsymbol{r}^{\boldsymbol{d}}\right): \boldsymbol{r} \in \Theta_{\boldsymbol{r}, \boldsymbol{\mu}}^{+}, \boldsymbol{r}^{\boldsymbol{d}}=f(\boldsymbol{r})\right\} .
$$

Coherency holds since this uncertainty set is a subset of the support of the random vector $\left(\tilde{\boldsymbol{r}}, \tilde{\boldsymbol{r}}^{\boldsymbol{d}}\right)$, see Remark 2.1. A risk-tolerant investor may want to move away from the minimum risk portfolio. This is achieved by appending an expected return constraint to the problem:

$$
\mathbb{E}\left[\tilde{r}_{p}\right]=\boldsymbol{w}^{T} \boldsymbol{\mu}+\left(\boldsymbol{w}^{\boldsymbol{d}}\right)^{T} \mathbb{E}[\max \{\mathbf{0}, \boldsymbol{a}+\mathbf{B} \tilde{\boldsymbol{r}}\}] \geq \mu_{\text {target }} .
$$

For any distribution of $\tilde{\boldsymbol{r}}$, we can evaluate the expected return of the options via sampling. Since sampling is impractical when the expected returns are ambiguous, one may alternatively use a conservative approximation of the return target constraint (40),

$$
\boldsymbol{w}^{T} \boldsymbol{\mu}+\left(\boldsymbol{w}^{\boldsymbol{d}}\right)^{T}(\max \{\mathbf{0}, \boldsymbol{a}+\mathbf{B} \boldsymbol{\mu}\}) \geq \mu_{\text {target }} .
$$

Indeed, (40) is less restrictive than (41) by Jensen's inequality. To account for the uncertainty in the estimated means, we can further robustify (41) as follows,

$$
\left.\begin{array}{ll}
\max _{\boldsymbol{q} \in \mathbb{R}^{m}} & \boldsymbol{\mu}^{T}\left(\boldsymbol{w}+\mathbf{B}^{T} \boldsymbol{q}\right)+\boldsymbol{a}^{T} \boldsymbol{q} \\
\text { subject to } & \mathbf{0} \leq \boldsymbol{q} \leq \boldsymbol{w}^{\boldsymbol{d}}
\end{array}\right\} \geq \mu_{\text {target }} \quad \forall \boldsymbol{\mu} \in \Theta_{\boldsymbol{\mu}}
$$


which is equivalent to

$$
\left.\begin{array}{ll}
\max _{\boldsymbol{q} \in \mathbb{R}^{m}} & \hat{\boldsymbol{\mu}}^{T}\left(\boldsymbol{w}+\mathbf{B}^{T} \boldsymbol{q}\right)-\kappa\left\|\boldsymbol{\Omega}^{1 / 2}\left(\boldsymbol{w}+\mathbf{B}^{T} \boldsymbol{q}\right)\right\|_{2}+\boldsymbol{a}^{T} \boldsymbol{q} \\
\text { subject to } & \mathbf{0} \leq \boldsymbol{q} \leq \boldsymbol{w}^{\boldsymbol{d}}
\end{array}\right\} \geq \mu_{\text {target }} .
$$

As a third alternative, the investor may wish to disregard the expected returns of the options altogether in the return target constraint. Taking into account the uncertainty in the estimated means, we thus obtain the second-order cone constraint

$$
\boldsymbol{w}^{T} \hat{\boldsymbol{\mu}}-\kappa\left\|\boldsymbol{\Omega}^{1 / 2} \boldsymbol{w}\right\|_{2} \geq \mu_{\text {target }},
$$

which is identical to (21). The advantages of this third approach are twofold.

Firstly, by omitting the options in the expected return constraint, we force the model to use the options for risk reduction and insurance only, but not for speculative reasons. Only the stocks are used to attain the prescribed expected return target. In light of the substantial risks involved in speculation with options, this might be attractive for risk-averse investors.

Secondly, the inclusion of an expected return constraint converts (35) to a mean-risk model [23], which minimizes a coherent downside risk measure, see Remark 2.1. However, Dert and Oldenkamp [19] and Lucas and Siegmann [28] have identified several pitfalls that may arise when using mean-downside risk models in the presence of highly asymmetric asset classes such as options and hedge funds. The particular problems that occur in the presence of options have been characterized as the Casino Effect: Mean-downside risk models typically choose portfolios which use the least amount of money that is necessary to satisfy the insurance constraint, whilst allocating the remaining money in the assets with the highest expected return. In our context, a combination of inexpensive stocks and put options will be used to satisfy the insurance constraint. Since call options are leveraged assets and have expected returns that increase with the strike price [17], the remaining wealth will therefore generally be invested in the call options with the highest strike prices available. The resulting portfolios have a high probability of small losses and a very low probability of high returns. Since the robust framework is typically used by risk-averse investors, the resulting portfolios are most likely in conflict with their risk preferences. It should be emphasized that the Casino Effect is characteristic for mean-downside risk models and not a side-effect of the robust portfolio optimization methodology. In order to alleviate its impact, Dert and Oldenkamp propose the use of several Value-at-Risk constraints to shape the distribution of terminal wealth. Lucas and Siegmann propose a modified risk measure that incorporates a quadratic penalty function to the expected losses. In all our numerical tests, we choose to exclude the expected option returns from the return target constraint. This will avoid betting on the options and thus mitigate the Casino Effect. As we will show in the next section, our numerical results indicate that the suggested portfolio model successfully reduces the downside risk and sustains high out-of-sample expected returns.

\section{Computational Results}

In Section 4.1 we investigate the optimal portfolio composition for different levels of riskaversion and illustrate the tradeoff between the weak non-inferiority guarantee and the strong insurance guarantee. In Section 4.2 we conduct several tests based on simulated data, while the tests in Section 4.3 are performed on the basis of real market data. In both 
sections, we compare the out-of-sample performance of the insured robust portfolios with that of the non-insured robust and classical mean-variance portfolios. The comparisons are based on the following performance measures: average yearly return, worst-case and best-case monthly returns, yearly variance, skewness, and Sharpe ratio [40]. All computations are performed using the $\mathrm{C}++$ interface of the MOSEK 5.0.0.105 conic optimization toolkit on a $2.0 \mathrm{GHz}$ Core 2 Duo machine running Linux Ubuntu 8.04. The details of the experiments are described in the next sections.

\subsection{Portfolio Composition and Tradeoff of Guarantees}

All experiments in this section are based on the $n=30$ stocks in the Dow 30 index. We assume that for each stock there are 40 put and 40 call options that mature in one year. The 40 strike prices of the put and call options for one particular stock are located at equidistant points between $70 \%$ and $130 \%$ of the stock's current price. In total, the market thus comprises 2400 options in addition to the 30 stocks.

In our first simulated backtests, we assume that the stock prices are governed by a multivariate geometric Brownian motion,

$$
\begin{gathered}
\frac{\mathrm{d} \tilde{S}_{t}^{i}}{\tilde{S}_{t}^{i}}=\mu_{i}^{c} \mathrm{~d} t+\sigma_{i}^{c} \mathrm{~d} \widetilde{W}_{t}^{i}, \quad i=1 \ldots n, \\
\mathbb{E}\left[\mathrm{d} \widetilde{W_{t}^{i}} \mathrm{~d} \widetilde{W}_{t}^{j}\right]=\rho_{i j}^{c} \mathrm{~d} t, \quad i, j=1 \ldots n,
\end{gathered}
$$

where $\tilde{S}^{i}$ denotes the price process of stock $i$ and $\widetilde{W}^{i}$ denotes a standard Wiener process. The continuous-time parameters $\mu_{i}^{c}, \sigma_{i}^{c}$, and $\rho_{i j}^{c}$ represent the drift rates, volatilities and correlation rates of the instantaneous stock returns, respectively. We calibrate this stochastic model to match the annualized means and covariances of the total returns of the Dow 30 stocks reported in Idzorek [25]. The transformation which maps the annualized parameters to the continuous-time parameters in (43) is described in [35, p. 345]. Furthermore, we assume that the risk-free rate amounts to $r_{f}=5 \%$ per annum and that the options are priced according to the Black-Scholes formula [11].

In the experiments of this section we do not allow short-selling of stocks. Furthermore, we assume that there is no parameter uncertainty. Therefore, we set $q=0$. In the first set of tests we solve problem (35) without an expected return constraint and without a portfolio insurance constraint. We determine the optimal portfolio allocations for increasing sizes of uncertainty sets parameterized by $p \in[0,1)$. The optimal portfolio weights are visualized in the top left panel of Figure 1, and the optimal conditional worst-case returns are displayed in the bottom left panel. For simplicity, we only report the total percentage of wealth allocated in stocks, calls, and put options, and provide no information about the individual asset allocations. All instances of problem (35) considered in this test were solved within less than 2 seconds, which manifests the tractability of the proposed model.

Figure 1 exhibits three different allocation regimes. For small values of $p$, the optimal portfolios are entirely invested in call options or a mixture of calls and stocks. This is a natural consequence of the leverage effect of the call options, which have a much higher return potential than the stocks when they mature in-the-money. As a result, the optimal conditional worst-case return is very high. Large investments in call options tend to be highly risky; this is reflected by a sudden decrease in call option allocation at threshold value $p \approx 7 \%$.

We also observe a regime which is entirely invested in stocks. Here, the risk is minimized through variance reduction by diversification, and no option hedging is involved. 
At higher uncertainty levels, there is a sudden shift to portfolios composed of stocks and put options. This transition takes place when the uncertainty set is large enough such that stock-only portfolios necessarily incur a loss in the worst case. The effect of the put options can be observed in the bottom left panel of Figure 1, which shows a constant worst-case return $\phi>1$ for higher uncertainty levels. Here, risk is not reduced through diversification. Instead, an aggressive portfolio insurance strategy is adopted using deep in-the-money put options. The put options are used to cut away the losses, and thus $\phi>1$. For high uncertainty levels, maximizing the conditional worst-case return amounts to maximizing the absolute insurance guarantee because the uncertainty set converges to the support of the returns, see Proposition 3.1.

The Black-Scholes market under consideration is arbitrage-free. An elementary arbitrage argument implies that the maximum guaranteed lower bound on the return of any portfolio is not larger than the risk-free return $\exp \left(r_{f} T\right)$. The conditional worst-case return in problem (35) is therefore bounded above by $\exp \left(r_{f} T\right)$ already for moderately sized uncertainty sets. This risk-free return can indeed be attained, al least approximately, by combining a stock and a put option on that stock with a very large strike price. Note that the put option matures in-the-money with high probability. Thus, the resulting portfolio pays off the strike price in most cases and is almost risk-free. Its conditional worst-case return is only slightly smaller than $\exp \left(r_{f} T\right.$ ) (for large uncertainty sets with $p \lesssim 1$ ). However, investing in an almost risk-free portfolio keeps the expected portfolio return fairly low, that is, close to the risk-free return.

In order to bypass this shortcoming, we impose an expected return constraint on the stock part of the portfolio with a target return of $8 \%$ per annum, see (42). The results of model (35) with an expected return constraint and without a portfolio insurance constraint are visualized on the right hand side of Figure 1. Most of the earlier conclusions remain valid, but there are a few differences. Because the stocks are needed to satisfy the return target, we now observe that all portfolios put a minimum weight of nearly $90 \%$ in stocks. For higher levels of uncertainty, the allocation in put options increases gradually when higher uncertainty is assigned to the returns.

The optimal conditional worst-case return smoothly degrades for increasing uncertainty levels and now drops below 1 . Here, we anticipate a loss in the worst-case. Recall that the (negative) conditional worst-case return can be interpreted as a risk measure, see Remark 2.1. In order to satisfy the expected return constraint, the optimal portfolios have to take higher risks than in the absence of an expected return constraint. As a result, the optimal conditional worst-case return is now lower (due to the higher risk) than before. This is a natural consequence of the risk-return tradeoff. For $p \gtrsim 90 \%$, the conditional worst-case return saturates at the worst-case return that can be guaranteed with certainty.

Next, we analyze the effects of the insurance constraint on the conditional worst-case return. To this end, we solve problem (35) for various insurance levels $\theta \in[0,1]$ and uncertainty levels $p \in[0,1)$, whilst still requiring the expected return to exceed $8 \%$. Figure 2 shows the conditional worst-case return as a function of $p$ and $\theta$.

For any fixed $p$, the conditional worst-case return monotonically decreases with $\theta$. Observe that this decrease is steeper for lower values of $p$. When the uncertainty set is small, the conditional worst-case return is relatively high. Therefore, the inclusion of the insurance guarantee has a significant impact due to the high insurance costs that are introduced. When the uncertainty set size is increased, the conditional worst-case return drops, and portfolio insurance needs to be provided for a lower worst-case portfolio return at an associated lower portfolio insurance cost.

When $\theta=1$, the portfolio is insured against dropping below the conditional worst-case 


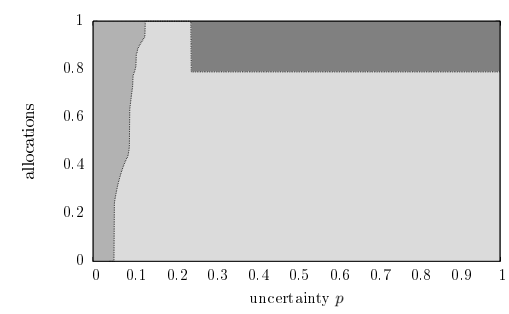

put options

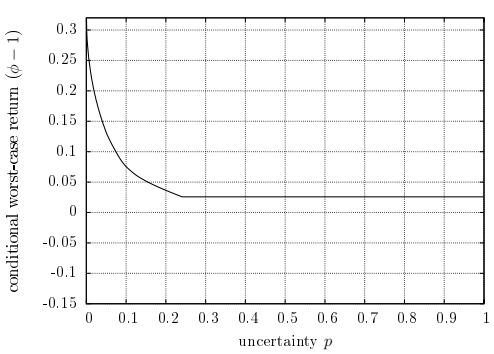

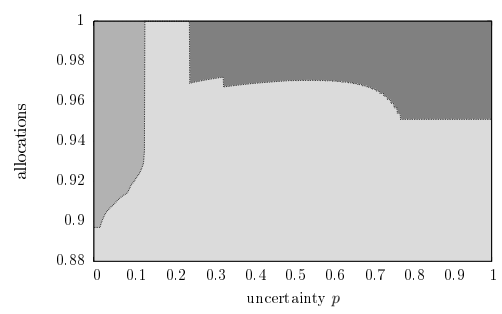

put options
call opt ions

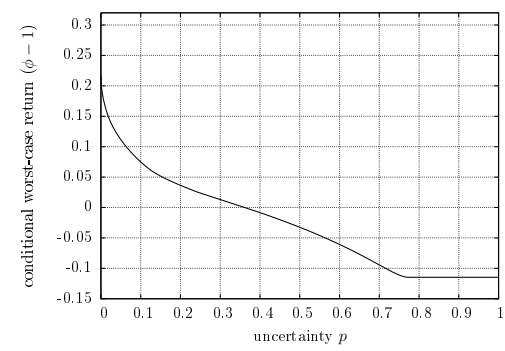

Figure 1: Visualization of the optimal portfolio allocations (top) and corresponding conditional worst-case returns (bottom), with (right) and without (left) an expected return constraint.

return. That is, the optimal portfolio provides the highest possible insurance guarantee that is still compatible with the expected return target. This optimal portfolio is independent of the size of the uncertainty set, and therefore the worst-case return is constant in $p$. For $p \gtrsim 80 \%$, the uncertainty set converges to the support of the returns, and the resulting optimal portfolio is independent of $\theta$, see Proposition 3.1. Note that if the expected return target is increased, then the guaranteed worst-case return for $\theta=1$ decreases. In fact, in order to satisfy the higher expected return constraint the cost of insurance has to be decreased. The cost of insurance can only be lowered by decreasing the allocation in put options, which implies a lower guaranteed worst-case return.

\subsection{Out-of-Sample Evaluation Using Simulated Prices}

A series of controlled experiments with simulated data help us to assess the performance of the proposed Insured Robust Portfolio Optimization (IRPO) model under different market conditions. We first generate price paths under a multivariate geometric Brownian motion model to reflect "normal" market conditions. Next, we use a multivariate jumpdiffusion process to simulate a volatile environment in which market crashes can occur. In both settings, we compare the performance of the IRPO model to that of the Robust Portfolio Optimization (RPO) model (19), and the classical Mean-Variance Optimization (MVO) model. The optimal Mvo portfolio is found by minimizing the variance of the portfolio return subject to an expected portfolio return constraint. In this case the estimated means and covariance matrix of the asset returns are used without taking parameter uncertainty into account. In Section 4.2.1 we describe the data simulation, the rolling-horizon backtest procedure, and the various performance measures that are used to compare the models. In Section 4.2.2 we discuss the test results. 


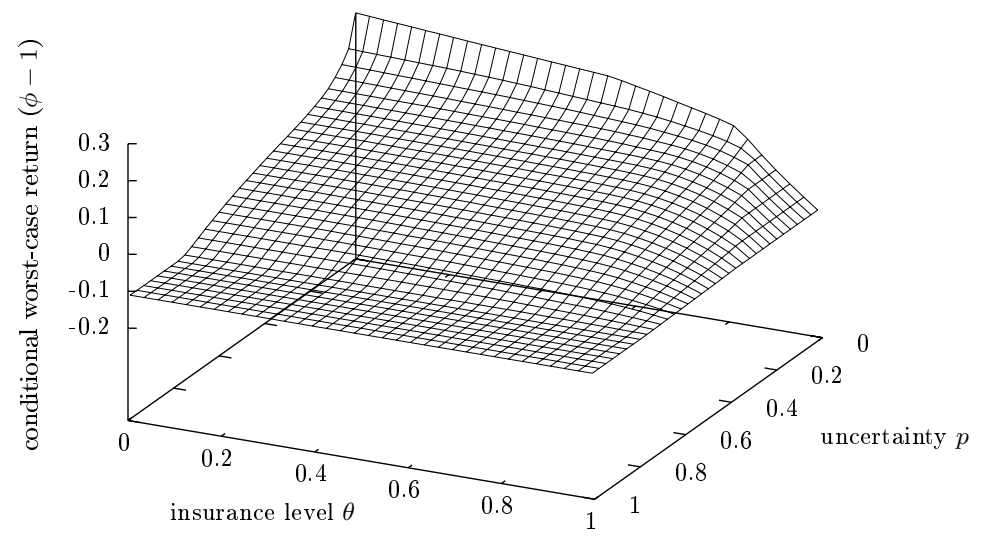

Figure 2: Tradeoff of weak and strong guarantees.

\subsubsection{Backtest Procedure and Evaluation}

The following experiments are again based on the stocks in the Dow 30 index. The first test series is aimed at assessing the performance of the models under "normal" market conditions. To this end, we assume that the stock prices are governed by the multivariate geometric Brownian motion described in (43).

We denote by $\tilde{\boldsymbol{r}}_{l}$ the vector of the asset returns over the interval $[(l-1) \Delta t, l \Delta t]$, where $\Delta t$ is set to one month (i.e., $\Delta t=1 / 12$ ) and $l \in \mathbb{N}$. By solving the stochastic differential equations (43), we find

$$
\tilde{r}_{l}^{i}=\exp \left[\left(\mu_{i}^{c}-\frac{\left(\sigma_{i}^{c}\right)^{2}}{2}\right) \Delta t+\tilde{\epsilon}_{l}^{i} \sqrt{\Delta t}\right], \quad i=1 \ldots n,
$$

where $\left\{\tilde{\boldsymbol{\epsilon}}_{l}\right\}_{l \in \mathbb{N}}$ are independent and identically normally distributed with zero mean and covariance matrix $\Sigma^{c} \in \mathbb{R}^{n \times n}$ with entries $\Sigma_{i j}^{c}=\rho_{i j}^{c} \sigma_{i}^{c} \sigma_{j}^{c}$ for $i, j=1 \ldots n$.

To evaluate the performance of the different portfolio models, we use the following rolling-horizon procedure:

1. Generate a time-series of $L$ monthly stock returns $\left\{\boldsymbol{r}_{l}\right\}_{l=1}^{L}$ using (44) and initialize the iteration counter at $l=E$. The number $E<L$ determines the size of a moving estimation window.

2. Calculate the sample mean $\hat{\boldsymbol{\mu}}_{l}$ and sample covariance matrix $\hat{\boldsymbol{\Sigma}}_{l}$ of the stock returns $\left\{\boldsymbol{r}_{l}\right\}_{l^{\prime}=l-E+1}^{l}$ in the current estimation window. We assume that there are 20 put and 20 call options available for each stock that expire after one month. The 20 strike prices of the options are assumed to scale with the underlying stock price: the proportionality factor ranges from $80 \%$ to $120 \%$ in steps of $2 \%{ }^{2}$

Next, convert the estimated monthly volatilies to continuous-time volatilities via the transformation in [35, p. 345] and calculate the option prices via the Black-

\footnotetext{
${ }^{2}$ This set of options is a reasonable proxy for the set available in reality. Depending on liquidity, there might be more or fewer options available, but the use of 20 strike prices oriented around the spot prices seems a good compromise.
} 


\begin{tabular}{ccccc|ccccc} 
Model $k$ & Type & $p$ & $q$ & $\theta$ & Model $k$ & Type & $p$ & $q$ & $\theta$ \\
\hline \hline 1 & MVO & - & - & - & & & & & \\
2 & RPO & 0.50 & 0.80 & - & 17 & IRPO & 0.70 & 0.80 & 0.00 \\
3 & RPO & 0.60 & 0.80 & - & 18 & IRPO & 0.70 & 0.80 & 0.70 \\
4 & RPO & 0.70 & 0.80 & - & 19 & IRPO & 0.70 & 0.80 & 0.80 \\
5 & RPO & 0.80 & 0.80 & - & 20 & IRPO & 0.70 & 0.80 & 0.90 \\
6 & RPO & 0.90 & 0.80 & - & 21 & IRPO & 0.70 & 0.80 & 0.99 \\
7 & IRPO & 0.50 & 0.80 & 0.00 & 22 & IRPO & 0.80 & 0.80 & 0.00 \\
8 & IRPO & 0.50 & 0.80 & 0.70 & 23 & IRPO & 0.80 & 0.80 & 0.70 \\
9 & IRPO & 0.50 & 0.80 & 0.80 & 24 & IRPO & 0.80 & 0.80 & 0.80 \\
10 & IRPO & 0.50 & 0.80 & 0.90 & 25 & IRPO & 0.80 & 0.80 & 0.90 \\
11 & IRPO & 0.50 & 0.80 & 0.99 & 26 & IRPO & 0.80 & 0.80 & 0.99 \\
12 & IRPO & 0.60 & 0.80 & 0.00 & 27 & IRPO & 0.90 & 0.80 & 0.00 \\
13 & IRPO & 0.60 & 0.80 & 0.70 & 28 & IRPO & 0.90 & 0.80 & 0.70 \\
14 & IRPO & 0.60 & 0.80 & 0.80 & 29 & IRPO & 0.90 & 0.80 & 0.80 \\
15 & IRPO & 0.60 & 0.80 & 0.90 & 30 & IRPO & 0.90 & 0.80 & 0.90 \\
16 & IRPO & 0.60 & 0.80 & 0.99 & 31 & IRPO & 0.90 & 0.80 & 0.99 \\
\hline
\end{tabular}

Table 1: Parameter settings of the portfolio models used in the backtests.

Scholes formula. ${ }^{3}$ For the IRPO model we then calculate the necessary option related data $\boldsymbol{a}_{l}$ and $\mathbf{B}_{l}$ defined in (25).

3. Determine the optimal portfolios $\left(\boldsymbol{w}_{l}^{k}, \boldsymbol{w}_{l}^{\boldsymbol{d}, k}\right)$ corresponding to the models $k=$ $1, \ldots, 31$ specified in Table 1 .

4. For strategy $k$, the portfolio return $r_{l+1}^{k}$ over the interval $[l \Delta t,(l+1) \Delta t]$ is given by:

$$
r_{l+1}^{k}=\left(\boldsymbol{w}_{l}^{k}\right)^{T} \boldsymbol{r}_{l+1}+\left(\max \left\{\mathbf{0}, \boldsymbol{a}_{l}+\mathbf{B}_{l} \boldsymbol{r}_{l+1}\right\}\right)^{T} \boldsymbol{w}_{l}^{\boldsymbol{d}, k} .
$$

Since $\boldsymbol{r}_{l+1}$ is outside of the estimation window, this constitutes an out-of-sample evaluation.

5. If $l<L-1$, then increment $l$ and go to step 2. Otherwise, terminate.

In all backtests we set $L=240$ and use an estimation window of size $E=120$. We set the risk-free rate to $r_{f}=5 \%$ per annum and the expected return target to $8 \%$ per annum. We allow short-selling of individual stocks up to $-20 \%$ and do not impose upper bounds on the portfolio weights.

The rolling-horizon procedure generates $L-E$ returns $\left\{r_{l}^{k}\right\}_{l=E+1}^{L}$ for our 31 portfolio strategies indexed by $k$. For each of these strategies we calculate the following performance measures: the out-of-sample mean, variance, skewness, Sharpe ratio, worst-case

\footnotetext{
${ }^{3}$ In reality, one would use option prices observed in the market instead of calculated ones. An empirical backtest based on real option price data is provided in Section 4.3.
} 
and best-case monthly return.

$$
\begin{aligned}
\hat{\mu}^{k} & =\frac{1}{L-E} \sum_{l=E+1}^{L} r_{l}^{k}, & & \text { (mean) } \\
\left(\hat{\sigma}^{2}\right)^{k} & =\frac{1}{L-E-1} \sum_{l=E+1}^{L}\left(r_{l}^{k}-\hat{\mu}^{k}\right)^{2}, & & \text { (variance) } \\
\hat{\gamma}^{k} & =\frac{1}{L-E} \sum_{l=E+1}^{L}\left(\left(r_{l}^{k}-\hat{\mu}^{k}\right) / \hat{\sigma}^{k}\right)^{3}, & & \text { (skewness) } \\
\widehat{S R}^{k} & =\frac{\hat{\mu}^{k}-r_{f}}{\hat{\sigma}^{k}}, & & \text { (Sharpe ratio) } \\
\hat{\underline{r}}^{k} & =\min \left\{r_{l}^{k}: E+1 \leq l \leq L\right\}, & & \text { (worst-case return) } \\
\hat{\bar{r}}^{k} & =\max \left\{r_{l}^{k}: E+1 \leq l \leq L\right\} . & & \text { (best-case return) }
\end{aligned}
$$

By assuming an initial wealth of 1 , we also calculate the final wealth $\hat{\omega}^{k}$ of strategy $k$ as follows

$$
\hat{\omega}^{k}=\prod_{t=E+1}^{L} r_{l}^{k} .
$$

We repeat the rolling-horizon procedure described above $R=300$ times with different random generator seeds and calculate averages of the performance measures. We also estimate the probability of the different portfolio strategies (with $k>1$ ) yielding a higher final wealth than the Markowitz strategy (with $k=1$ ) by counting the simulation runs in which this outperformance is observed. Finally, we compute the excess return of any strategy $k$ relative to the Markowitz strategy, $\hat{\omega}^{k} / \hat{\omega}^{1}-1$, averaged over all simulation runs.

A property of the geometric Brownian motion price process is that there are almost surely no discontinuities in the price paths. In reality, rare events such as market crashes can occur, and therefore the Jump-Diffusion model introduced by Merton [33] may be more suitable to describe real price movements. Under Merton's Jump-Diffusion model, the stock prices are governed by the stochastic differential equations

$$
\begin{array}{ll}
\frac{\mathrm{d} \tilde{S}_{t}^{i}}{\tilde{S}_{t}^{i}}=\left(\mu_{i}^{c}-\lambda^{c} \eta\right) \mathrm{d} t+\sigma_{i}^{c} \mathrm{~d} \widetilde{W}_{t}^{i}+\mathrm{d} \sum_{j=1}^{\tilde{N}_{t}}\left(\tilde{Y}_{j}-1\right), & i=1 \ldots n, \\
\mathbb{E}\left[\mathrm{d} \widetilde{W}_{t}^{i} \mathrm{~d} \widetilde{W}_{t}^{j}\right]=\rho_{i j}^{c} \mathrm{~d} t, & i, j=1 \ldots n,
\end{array}
$$

where $\tilde{N}$ is a Poisson process with arrival intensity $\lambda^{c}$, and $\left\{\tilde{Y}_{j}\right\}_{j \in \mathbb{N}}$ is a sequence of independent identically distributed nonnegative random variables. $\tilde{N}_{t}$ denotes the number of jumps, or market crashes, between 0 and time $t$, while the $\tilde{Y}_{j}$ represent the relative price changes when such crashes occur. $\widetilde{W}_{t}$ and $\tilde{N}_{t}$ are assumed to be independent.

For simplicity, we assume that all stock prices jump at the same time. Moreover, instead of making the jump sizes stochastic, as in the general formulation above, we assume that all prices experience a deterministic relative change of $\eta=-15 \%$ when a crash occurs. We set $\lambda^{c}=2$, indicating that on average there are two crashes per year. Solving the stochastic differential equations (45) we obtain the following expression for 
the stock returns

$$
\tilde{r}_{l}^{i}=\exp \left[\left(\mu_{i}^{c}-\frac{\left(\sigma_{i}^{c}\right)^{2}}{2}-\lambda^{c} \eta\right) \Delta t+\tilde{\epsilon}_{l}^{i} \sqrt{\Delta t}\right] \prod_{j=\tilde{N}_{(l-1) \Delta t}+1}^{\tilde{N}_{l \Delta t}} Y_{j}, \quad i=1 \ldots n
$$

where $\tilde{N}_{t}$ follows a Poisson distribution with parameter $\lambda^{c} \Delta t$ and $Y_{j}=e^{\eta}$ for all $j$. We now repeat the previously described rolling-horizon backtest by using (46) instead of (44).

\subsubsection{Discussion of Results}

The results of our simulated backtests based on the geometric Brownian motion model are summarized in Table 3.

In comparison with the nominal MVO portfolio, we observe that the RPO portfolios exhibit a significantly higher average return at the cost of a relatively small increase in variance. This is also reflected by the Sharpe ratio values, which are higher than that of the Mvo portfolio for all levels of $p$. When $p$ increases, we notice a slight decrease in variance and expected return because the portfolios become more conservative. We see that the non-insured RPO portfolios outperform the MVO portfolio with probability $75 \%$. This indicates that taking the uncertainty of the mean estimates into account results in a considerable improvement of out-of-sample performance.

Next, we assess the performance of the IRPO portfolios. For a fixed insurance level $\theta$, we observe that the worst-case monthly return (Min) increases with $p$. In most cases, it also increases with $\theta$ for fixed $p$. However, this is not always the case. At $p=80 \%$, for instance, the worst-case return for $\theta=90 \%$ is higher than for $\theta=99 \%$. The reason for this is that a large portion of wealth is allocated to the options in order to satisfy the high insurance demands. Because there are no price jumps, these options have a low probability to mature in-the-money. The options have a noticeable effect on the skewness of the portfolio returns, which increases with $p$ and $\theta$. This is because the put options are effectively cutting away the losses and therefore cause the portfolio return distribution to be positively skewed.

Finally, for all tested values of $p$ and $\theta$, the IRPO portfolios accumulate a higher final wealth than the nominal MVo portfolio in about $65 \%$ of the cases. In terms of Sharpe ratio, the IRPO portfolios perform comparable to the RPO portfolios. However, the noninsured RPO portfolios have an increased expected return and a higher probability of outperforming the nominal MVO portfolio in terms of realized wealth. Note that, although the IRPO portfolios have a lower probability of outperforming the MVO portfolio, they achieve higher excess returns than the RPO portfolios because the options help preserve wealth over time. We conclude that under normal market conditions the non-insured RPO model seems to generate the most attractive out-of-sample results.

The results of our simulated backtests based on Merton's jump diffusion model are summarized in Table 4. The following discussion highlights the differences to the results obtained using the geometric Brownian motion model.

The RPO portfolios still have a significant probability of outperforming the MVo portfolio in terms of realized wealth. Due to the crashes, however, this probability now decreases to $65 \%$ (as opposed to $75 \%$ in the absence of crashes). Notice that the worstcase monthly returns of the RPO portfolios are of the same order of magnitude as those of the MVO portfolio. We also observe that the realized returns for the RPO and MVO portfolios are highly negatively skewed because of the downward jumps of the prices. 
The IRPO portfolios have an increased expected return and lower variance with respect to the MVo portfolio for all tested values of $p$ and $\theta$. This is also reflected by an improvement in Sharpe ratio, which for $p=60 \%$ and $\theta=99 \%$ is $60 \%$ higher than that of the MVO portfolio. The IRPO portfolios exhibit increased skewness relative to the MVO and RPO portfolios. The skewness of the IRPO portfolios becomes positive for values of $p \geq 80 \%$ and $\theta=99 \%$. The worst-case return gradually improves with increasing values of $p$ and $\theta$, and for $p=90 \%$ the worst-case is $50 \%$ higher than that of the nominal MVO portfolio. Finally, the IRPO portfolios achieve a higher realized wealth than the MVO portfolio in about $77 \%$ of the simulation runs. Notice also that the excess returns monotonically increase with $\theta$. The increase in realized wealth is due to the option insurance which helps preserve wealth during market crashes. In contrast, the crashes cause large losses of wealth to the MVO and RPO portfolios.

In conclusion, the simulated tests indicate that the IRPO model has advantages over the MVO and RPO models when the market exhibits jumps. It typically results in a higher realized wealth and Sharpe ratio.

\subsection{Out-of-Sample Evaluation Using Real Market Prices}

Simulated stock and option prices may give an unrealistic view of how our portfolio strategies perform in reality due to the following reasons. Firstly, it is known that real stock returns are not serially independent and identically distributed. Secondly, real option prices deviate from those obtained via the Black-Scholes formula by using historical volatilities. Finally, we are restricted to invest in the options traded in the market, and our assumption about the range of available strike prices may not hold.

Therefore, we now evaluate the portfolio strategies under the same rolling-horizon procedure described in the previous section but with real stock and option prices. Historical stock and option prices are obtained from the OptionMetrics IvyDB database, which is one of the most complete sources of historical option data available. We limit ourselves to the equity indices shown in Table 2. These indices were chosen because they have the most complete time-series in the database. As before, we rebalance on a monthly basis, and at every rebalancing date we consider all available European put and call options that expire in one month. ${ }^{4}$ Because the IRPO strategy is long in options, we use the highest option ask prices to make sure that we could have acquired the options at the specified prices.

The time-series covers the period from 18/01/1996 until 18/09/2008. We use an estimation window of 15 months. ${ }^{5}$ Moreover, we allow short-selling in every equity index up to $-20 \%$ of total portfolio value but impose no upper bounds on the weights. The target expected return is set to $8 \%$ per annum. The range of tested $p$ and $\theta$ values is the same as in the previous section, see Table 1.

\subsubsection{Discussion of Results}

The results of the backtests based on real market prices are given in Table 5. Similar to the out-of-sample results based on simulated prices, the RPO portfolios produce higher expected returns than the nominal MVo model, while their Sharpe ratios are more than twice as large as that of the MVO portfolio for all tested values of $p$.

\footnotetext{
${ }^{4}$ In order to avoid the use of erroneous option data, we only selected those options for which the implied volatility was supplied and which had a bid and ask price greater than 0 . We found that this procedure allowed us to filter out incorrect entries.

${ }^{5}$ Different estimation windows yielded slightly different out-of-sample results. However, the general conclusions are independent of the choice of the estimation window.
} 


\begin{tabular}{ll} 
Ticker & Name \\
\hline \hline XMI & AMEX Major Market Index \\
SPX & S\&P 500 Index \\
MID & S\&P Midcap 400 Index \\
SML & S\&P Smallcap 600 Index \\
RUT & Russell 2000 Index \\
NDX & NASDAQ 100 Index \\
\hline
\end{tabular}

Table 2: Equity indices used in the historical backtest.

The IRPO portfolios also outperform the MVO portfolio in terms of expected return and Sharpe ratio for all values of $p$ and $\theta$. However, compared to the RPO portfolios, they have a slightly lower expected return on average. This decrease in expected return is due to the cost of insurance. We also observe that the IRPO portfolios have smaller variance than the RPO portfolios for all tested parameter settings. On average the IRPO portfolios also produce slightly higher Sharpe ratios than the RPO portfolios.

In Figure 3 we plot the cumulated wealth over time of the MVO portfolio, an RPO portfolio with $p=50 \%$, an IRPO portfolio with $p=50 \%$ and $\theta=70 \%$, and an IRPO portfolio with $p=50 \%$ and $\theta=99 \%$. The IRPO portfolio with $\theta=70 \%$ performed better than the MVO and RPO portfolios. However, we emphasize that the performance of the IRPO model is highly dependent on the values chosen for $p$ and $\theta$. For example, it can be observed that with $p=50 \%$ and $\theta=99 \%$ the IRPO portfolio is outperformed by the RPO portfolio due to the high cost of insurance.

For all tested parameters values, the IRPO model yields a higher worst-case monthly return than the RPO model and a significant increase in skewness for levels of $p \geq 60 \%$. The worst-case return monotonically increases with $p$. However, it is not always increasing in $\theta$. High insurance levels of $\theta \gtrsim 90 \%$ lead to large investments in put options which expire worthless with high probability. This is also reflected by a significant drop in expected return and an associated decrease in Sharpe ratio.

The reasons for this are twofold. Firstly, the strong insurance guarantees are more expensive in reality than in the simulations. This is because the Black-Scholes formula underestimates the prices of far out-of-the-money put options when historical volatilies are used. Secondly, we are limited to invest in the options that are traded in the market and are therefore unable to invest in options with strike prices that would have resulted in better portfolios.

To conclude, we note that for this particular data set the RPO and IRPO portfolios systematically outperform the nominal MVO portfolio in terms of expected return and Sharpe ratio. On average the RPO portfolios achieve higher expected returns than the IRPO portfolios, whereas the IRPO portfolios obtain slightly higher average Sharpe ratios. We also conclude that the performance of the IRPO model is highly dependent on the chosen values of $p$ and $\theta$. The insurance levels should therefore be tuned to market behavior. Higher insurance levels can help preserve the accumulated portfolio wealth when the market is volatile and experiences jumps. Lower insurance levels are preferable in less volatile periods since unnecessary insurance costs are avoided.

\section{Conclusions}

In this paper, we extend robust portfolio optimization to accommodate options. Moreover, we show how the options can be used to provide strong insurance guarantees, which 


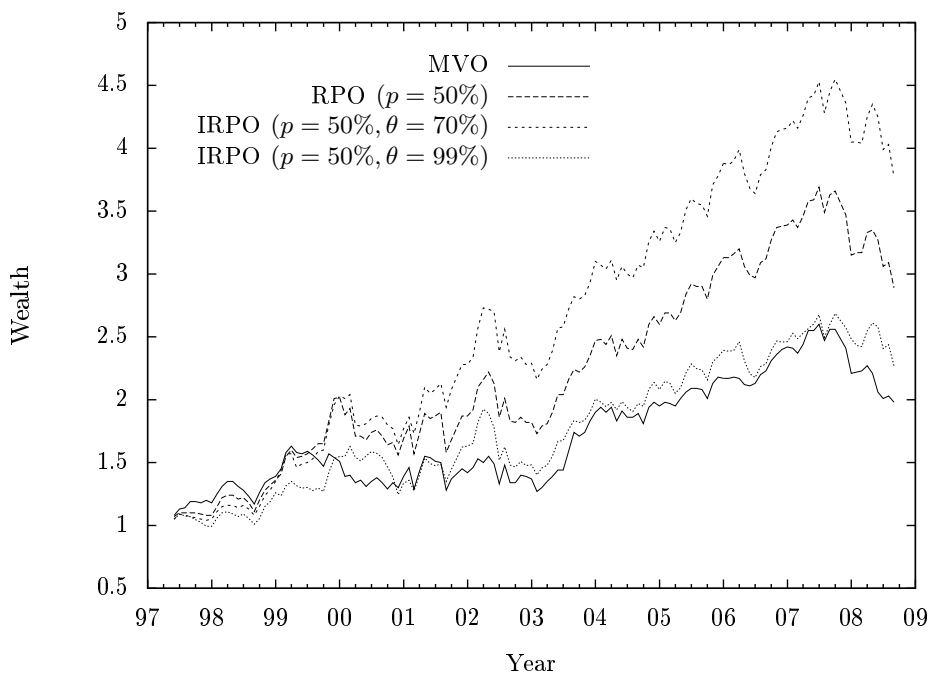

Figure 3: Cumulated return of the MVO, RPO, and IRPO portfolios using monthly rebalancing between $19 / 06 / 1997$ and $18 / 09 / 2008$.

also hold when the stock returns are realized outside of the prescribed uncertainty set. Using conic and linear duality, we reformulate the problem as a convex second-order cone program, which is scalable in the amount of stocks and options and can be solved efficiently with standard optimization packages. The proposed methodology can be applied to a wide range of uncertainty sets and can therefore be seen as a generic extension to the robust portfolio optimization framework.

We first perform backtests on simulated data, in which the asset prices reflect normal market conditions as well as market crashes. In both cases the option premia are calculated using the standard Black-Scholes model. The simulated results indicate that the insured robust portfolios have lower expected returns than the non-insured robust portfolios under normal market conditions but have clear advantages with respect to Sharpe ratio, expected return, as well as cumulative wealth, when the prices experience jumps.

Since the Black-Scholes prices might not reflect realistic option premia, we also perform backtests on historical data. We observe that on average the RPO portfolios achieve higher expected returns than the IRPO portfolios, whereas the IRPO portfolios obtain higher Sharpe ratios. The results also indicate that the performance of the IRPO model is highly dependent on the values chosen for $p$ and $\theta$. When the insurance level is set too high, the cost of insurance causes the performance to deteriorate. Therefore, the level of insurance should be tuned to the market; to preserve wealth, higher insurance levels can benefit the portfolio when the market is volatile and experiences jumps. Lower insurance levels are preferable in less volatile periods since unnecessary insurance costs are avoided.

\section{References}

[1] D. Ahn, J. Boudoukh, M. Richardson, and R. F. Whitelaw. Optimal risk management using options. The Journal of Finance, 54(1):359-375, 1999. 
[2] F. Alizadeh and D. Goldfarb. Second-order cone programming. Mathematical Programming, 95(1):3-51, 2003.

[3] P. Artzner, F. Delbaen, J. Eber, and D. Heath. Coherent measures of risk. Mathematical Finance, 9(3):203-228, 1999.

[4] A. Ben-Tal, S. Boyd, and A. Nemirovski. Extending scope of robust optimization: Comprehensive robust counterparts of uncertain problems. Math. Program., Ser. B, 107:63-89, 2006.

[5] A. Ben-Tal, T. Margalit, and A. Nemirovski. Robust modeling of multi-stage portfolio problems. In: Frenk, H., Roos, K., Terlaky, T., Zhang, S. (Eds.), High performance optimization, pages $303-328,2000$.

[6] A. Ben-Tal and A. Nemirovski. Robust convex optimization. Mathematics of Operations Research, 23(4):769-805, 1998.

[7] A. Ben-Tal and A. Nemirovski. Robust solutions of uncertain linear programs. Operations Research Letters, 25(1):1-13, 1999.

[8] D. Bertsimas and D.B. Brown. Constructing uncertainty sets for robust linear optimization. Operations Research, 57(6):1483-1495, 2009.

[9] D. Bertsimas and D. Pachamanova. Robust multiperiod portfolio management in the presence of transaction costs. Computers \&3 Operations Research, 35(1):3-17, 2008 .

[10] F. Black and R. Litterman. Asset allocation: Combining investor views with market equilibrium: Technical report. 1990.

[11] F. Black and M. S. Scholes. The pricing of options and corporate liabilities. Journal of Political Economy, 81(3):637-54, 1973.

[12] M. Broadie. Computing efficient frontiers using estimated parameters. Annals of Operations Research, 45(1):21-58, 1993.

[13] S. Ceria and R. Stubbs. Incorporating estimation errors into portfolio selection: Robust portfolio construction. Journal of Asset Management, 7(2):109-127, 2006.

[14] V. K. Chopra. Improving optimization. Journal of Investing, 2(3):51-59, 1993.

[15] V. K. Chopra, C. R. Hensel, and A. L. Turner. Massaging mean-variance inputs: Returns from alternative investment strategies in the 1980s. Management Science, 39(7):845-855, 1993.

[16] V. K. Chopra and W. T. Ziemba. The effect of errors in means, variances and covariances on optimal portfolio choice. Journal of Portfolio Management, 19(2):611, 1993.

[17] J. Coval and T. Shumway. Expected option returns. The Journal of Finance, 56(3):983 - 1009, 2002.

[18] V. DeMiguel and F. J. Nogales. Portfolio selection with robust estimation. Operations Research, 57(3):560-577, 2009. 
[19] C. Dert and B. Oldenkamp. Optimal guaranteed return portfolios and the casino effect. Operations Research, 48(5):768-775, 2000.

[20] L. El Ghaoui, M. Oks, and F. Outstry. Worst-case value-at-risk and robust portfolio optimization: A conic programming approach. Operations Research, 51(4):543-556, 2003.

[21] D. Fabozzi, P. Kolm, and Pachamanova D. Robust Portfolio Optimization and Management. Wiley, 2007.

[22] D. Goldfarb and G. Iyengar. Robust portfolio selection problems. Mathematics of Operations Research, 28(1):1-38, 2003.

[23] W.V. Harlow. Asset allocation in a downside-risk framework. Financial analysts journal, 47(5):28-40, 1991.

[24] M. Howe, B. Rustem, and M. Selby. Minimax hedging strategy. Computational Economics, 7(4):245-275, 1994.

[25] T. Idzorek. A step-by-step guide to the black-litterman model. Technical report, Duke University, 2002.

[26] R. Jagannathan and T. Ma. Risk reduction in large portfolios: Why imposing the wrong constraints helps. Journal of Finance, 58(4):1651-1684, 2003.

[27] M. S. Lobo, L. Vandenberghe, S. Boyd, and H. Lebret. Applications of second-order cone programming. Linear Algebra and its Applications, 284(1):193-228, 1998.

[28] A. Lucas and A. Siegmann. The effect of shortfall as a risk measure for portfolios with hedge funds. Journal of Business Finance \& Accounting, 35(1-2):200-226, 2008.

[29] D. G. Luenberger. Investment Science. Oxford University Press, Madison Avenue, New York, 1998.

[30] F. Lutgens, S. Sturm, and A. Kolen. Robust one-period option hedging. Operations Research, 54(6):1051-1062, 2006.

[31] L. G. MacMillan. Options as a strategic investment. Prentice Hall, 1992.

[32] H. Markowitz. Portfolio selection. Journal of Finance, 7(1):77-91, 1952.

[33] R. C. Merton. Option pricing when underlying stock returns are discontinuous. Journal of Financial Economics, 3(1-2):125-144, 1976.

[34] R. C. Merton. On estimating the expected return on the market: an exploratory investigation. Journal of Financial Economics, 8(4):323-361, 1980.

[35] A. Meucci. Risk and Asset Allocation. Springer, Berlin, 2005.

[36] R. O. Michaud. Efficient Asset Management: A Practical Guide to Stock Portfolio Management and Asset Allocation. Oxford University Press, 2001.

[37] K. Natarajan, D. Pachamanova, and M. Sim. Incorporating asymmetric distributional information in robust value-at-risk optimization. Management Science, 54(3):573-585, 2008. 
[38] B. Rustem, R. G. Becker, and W. Marty. Robust min-max portfolio strategies for rival forecast and risk scenarios. Journal of Economic Dynamics and Control, 24(11-12):1591-1621, 2000.

[39] B. Rustem and M. Howe. Algorithms for Worst-Case Design and Applications to Risk Management. Princeton University Press, 2002.

[40] W. F. Sharpe. Mutual fund performance. The Journal of Business, 39(1):119-138, 1966.

[41] R. H. Tütüncü and M. Koenig. Robust asset allocation. Annals of Operations Research, 132(1-4):157-187, 2004. 


\section{A Appendix}

\section{A.1 Notational Reference Table}

$\begin{array}{ll}n & \text { Number of stocks } \\ m & \text { Number of options } \\ \tilde{r}_{p} & \text { Total portfolio return } \\ r_{f} & \text { Risk-free rate } \\ \boldsymbol{w}, \boldsymbol{w}^{\boldsymbol{d}} & \text { Weights of the stocks and options, respectively } \\ \mathbf{1} & \text { Vector of ones } \\ \boldsymbol{l}, \boldsymbol{u} & \text { Lower and upper bounds on the weights of the stocks } \\ \tilde{\boldsymbol{r}}, \tilde{\boldsymbol{r}}^{\boldsymbol{d}} & \text { Total stock and option returns, respectively } \\ \boldsymbol{\mu}, \boldsymbol{\Sigma} & \text { Mean vector and covariance matrix of } \tilde{\boldsymbol{r}}, \text { respectively } \\ \hat{\boldsymbol{\mu}}, \hat{\boldsymbol{\Sigma}} & \text { Sample mean and sample covariance matrix of } \tilde{\boldsymbol{r}}, \text { respectively } \\ \boldsymbol{\Lambda} & \text { Covariance matrix of } \hat{\boldsymbol{\mu}} \\ \boldsymbol{\Omega} & \text { Modified covariance matrix of } \hat{\boldsymbol{\mu}} \\ \lambda & \text { Risk-aversion parameter } \\ \mu_{\mathrm{target}} & \text { Portfolio return target } \\ p, q & \text { Probabilities of } \tilde{\boldsymbol{r}} \text { and } \hat{\boldsymbol{\mu}} \text { to be realized within their respective } \\ \Theta_{\boldsymbol{r}} & \text { uncertainty sets, respectively } \\ \Theta_{\boldsymbol{r}}^{+} & \text {Uncertainty set for } \tilde{\boldsymbol{r}} \\ \Theta_{\boldsymbol{\mu}} & \text { Uncertainty set for } \tilde{\boldsymbol{r}} \text { including support infomation } \\ \Theta_{\boldsymbol{r}, \boldsymbol{\mu}}^{+} & \text {Uncertainty set for } \boldsymbol{\mu} \\ \delta, \kappa & \text { Uncertainty set for } \tilde{\boldsymbol{r}} \text { and } \boldsymbol{\mu} \text { including support infomation } \\ \phi & \text { Size parameters for the uncertainty sets } \Theta_{\boldsymbol{r}, \boldsymbol{\mu}}^{+} \text {and } \Theta_{\boldsymbol{\mu}}, \text { respectively } \\ \theta & \text { Conditional worst-case portfolio return } \\ T & \text { Insurance level } \\ \tilde{S}_{t}^{i}, i=1, \ldots, n & \text { End of investment horizon } \\ \widetilde{W}^{i}, i=1, \ldots, n & \text { Price of stock } i \text { at time } t \\ \tilde{N} & \text { Standard Wiener processes } \\ \lambda^{c} & \text { Poisson process } \\ \eta & \text { Arrival intensity } \\ \mu^{c}, \sigma^{c}, \rho^{c} & \text { Relative price change during crash } \\ L & \text { Instantaneous drifts, volatilities and correlation rates, respectively } \\ E & \text { Size of the time-series } \\ K_{i}, i=1, \ldots, m & \text { Size of the estimation window } \\ P_{i}, C_{i}, i=1, \ldots, m & \text { Strike price of option } i \\ \boldsymbol{a}, \mathbf{B} & \text { Price of option } i \text { if it is a call } / \text { put option } \\ f & \text { Parameters of function } f \\ & \text { Function relating } \tilde{\boldsymbol{r}} \text { and } \tilde{\boldsymbol{r}} \boldsymbol{d} \\ & \end{array}$

\section{A.2 Proof of Theorem A.1}

Theorem A.1 For $\Theta_{\boldsymbol{\mu}}$ defined as in (12), and $\Theta_{\boldsymbol{\Sigma}}=\{\hat{\boldsymbol{\Sigma}}\}$, problem (8) is equivalent to the following second-order cone program,

$$
\max _{\boldsymbol{w} \in \mathbb{R}^{n}}\left\{\boldsymbol{w}^{T} \hat{\boldsymbol{\mu}}-\kappa\left\|\boldsymbol{\Omega}^{1 / 2} \boldsymbol{w}\right\|_{2}-\delta\left\|\hat{\boldsymbol{\Sigma}}^{1 / 2} \boldsymbol{w}\right\|_{2} \mid \boldsymbol{w}^{T} \mathbf{1}=1, \boldsymbol{l} \leq \boldsymbol{w} \leq \boldsymbol{u}\right\},
$$

where

$$
\boldsymbol{\Omega}=\boldsymbol{\Lambda}-\frac{1}{\mathbf{1}^{T} \boldsymbol{\Lambda} \mathbf{1}} \boldsymbol{\Lambda} \mathbf{1} \mathbf{1}^{T} \boldsymbol{\Lambda}
$$


Proof Because $\Theta_{\Sigma}$ is a singleton, it is clear that problem (8) is equivalent to

$$
\max _{\boldsymbol{w} \in \mathbb{R}^{n}}\left\{\min _{\boldsymbol{\mu} \in \Theta_{\boldsymbol{\mu}}} \boldsymbol{w}^{T} \boldsymbol{\mu}-\delta\left\|\hat{\boldsymbol{\Sigma}}^{1 / 2} \boldsymbol{w}\right\|_{2} \mid \boldsymbol{w}^{T} \mathbf{1}=1, \boldsymbol{l} \leq \boldsymbol{w} \leq \boldsymbol{u}\right\} .
$$

When $\kappa=0$, the claim is obviously true. In the rest of the proof we thus assume that $\kappa>0$. Using the definition of the uncertainty set $\Theta_{\boldsymbol{\mu}}$, the inner minimization problem in $(47)$ can be rewritten as

$$
\begin{aligned}
& \min _{\boldsymbol{\mu} \in \mathbb{R}^{n}} \quad \boldsymbol{w}^{T} \boldsymbol{\mu} \\
& \text { subject to }\left\|\boldsymbol{\Lambda}^{-1 / 2}(\boldsymbol{\mu}-\hat{\boldsymbol{\mu}})\right\|_{2} \leq \kappa \\
& \mathbf{1}^{T}(\boldsymbol{\mu}-\hat{\boldsymbol{\mu}})=0 .
\end{aligned}
$$

For any fixed portfolio $\boldsymbol{w}$, problem (48) represents a second-order cone program. We proceed by dualizing (48). After a few minor simplification steps, we obtain the dual problem

$$
\max _{q \in \mathbb{R}} \boldsymbol{w}^{T} \hat{\boldsymbol{\mu}}-\kappa\left\|\boldsymbol{\Lambda}^{1 / 2}(\boldsymbol{w}-q \mathbf{1})\right\| .
$$

Strong conic duality holds since the primal problem (47) is strictly feasible for $\kappa>0$. Thus, both the primal and dual problems (47) and (48) are feasible and share the same objective values at optimality. Since $\kappa>0$, the optimal dual solution is given by

$$
q^{*}=\underset{q \in \mathbb{R}}{\operatorname{argmin}}\left\|\boldsymbol{\Lambda}^{1 / 2}(\boldsymbol{w}-q \mathbf{1})\right\|=\frac{\boldsymbol{w}^{T} \boldsymbol{\Lambda} \mathbf{1}}{\mathbf{1}^{T} \boldsymbol{\Lambda} \mathbf{1}} .
$$

By substituting $q^{*}$ into (49) we obtain the optimal value of (48), which amounts to

$$
\boldsymbol{w}^{T} \hat{\boldsymbol{\mu}}-\kappa\left\|\boldsymbol{\Omega}^{1 / 2} \boldsymbol{w}\right\|_{2}
$$

We can now substitute (50) into (47) to obtain the postulated result. 


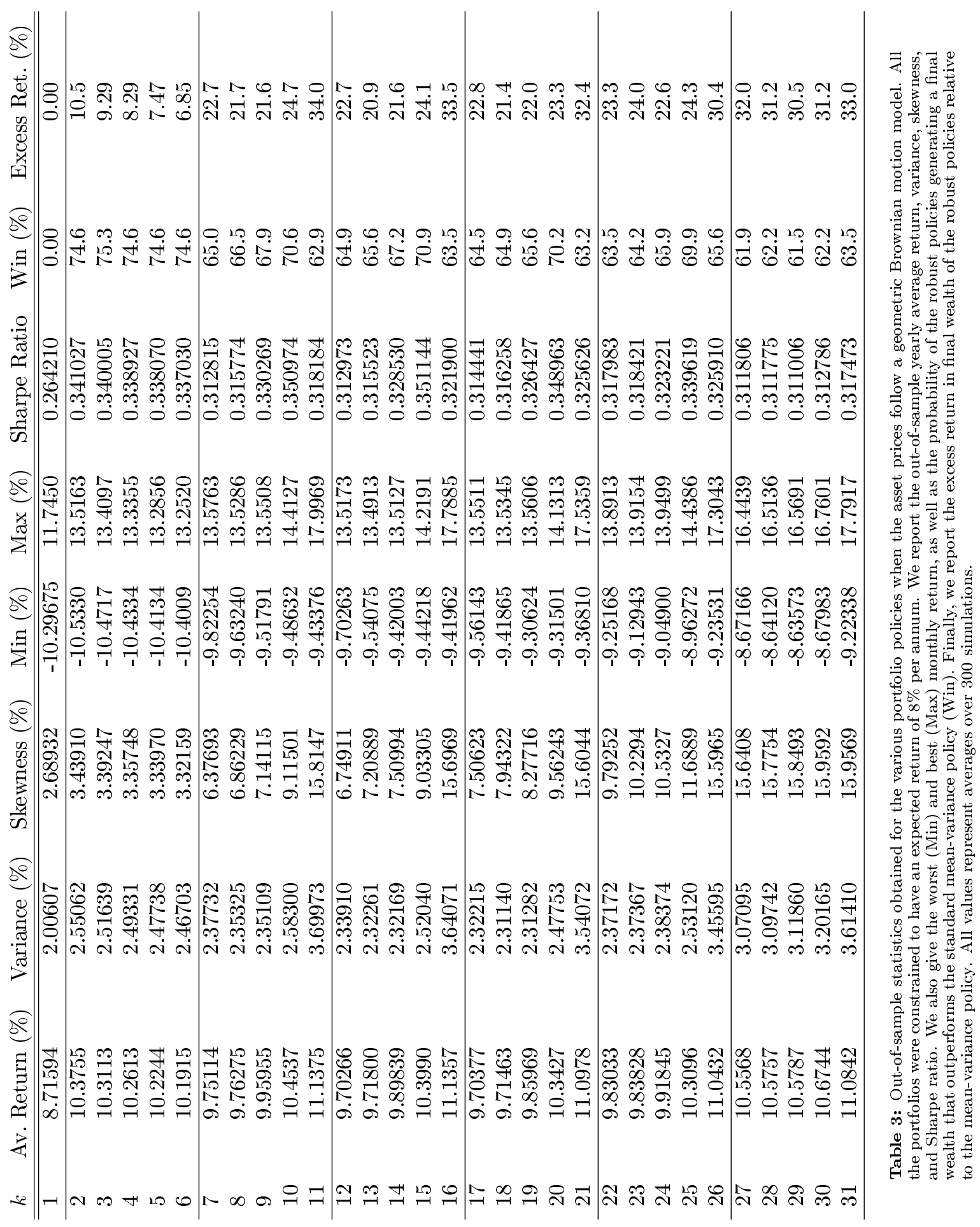




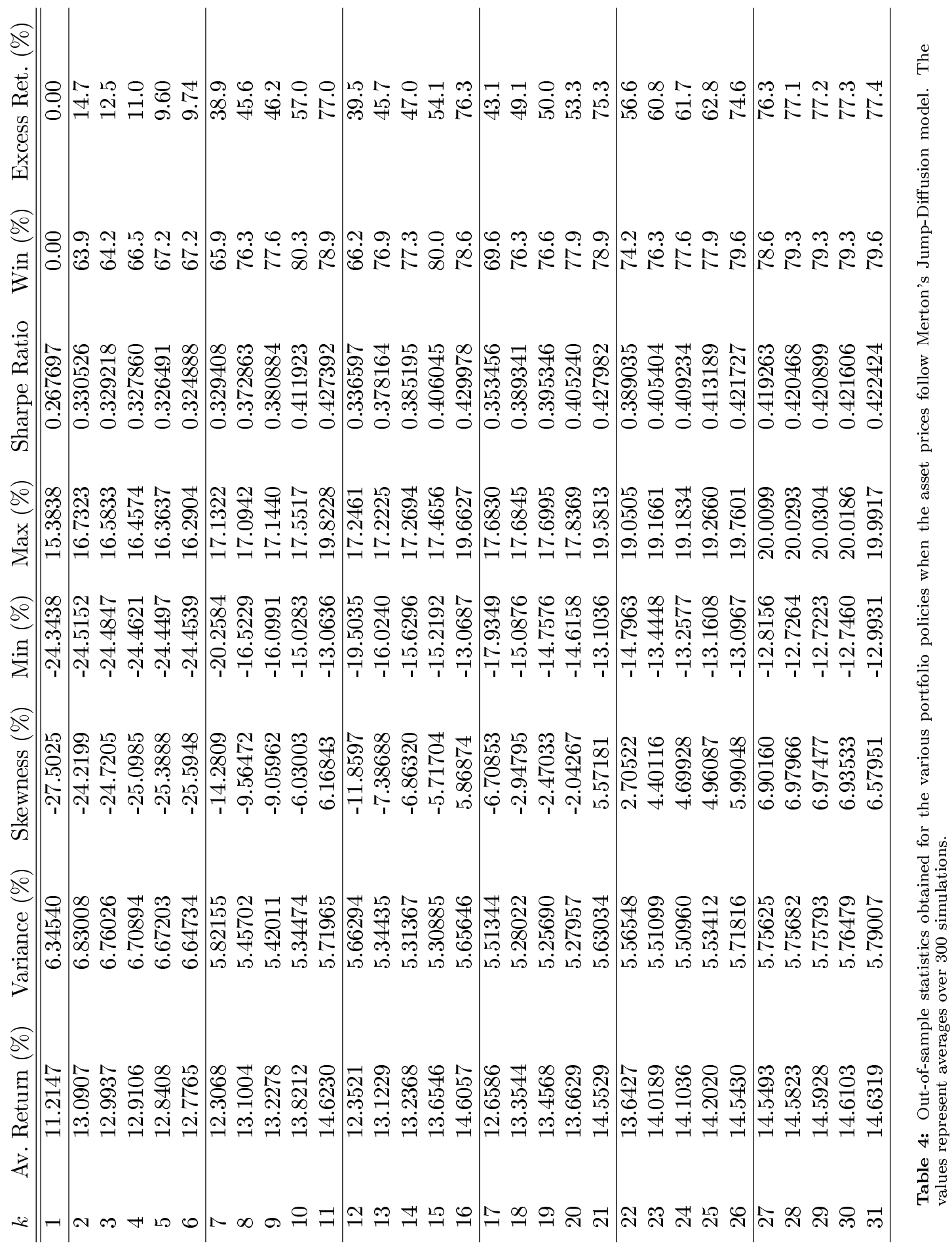




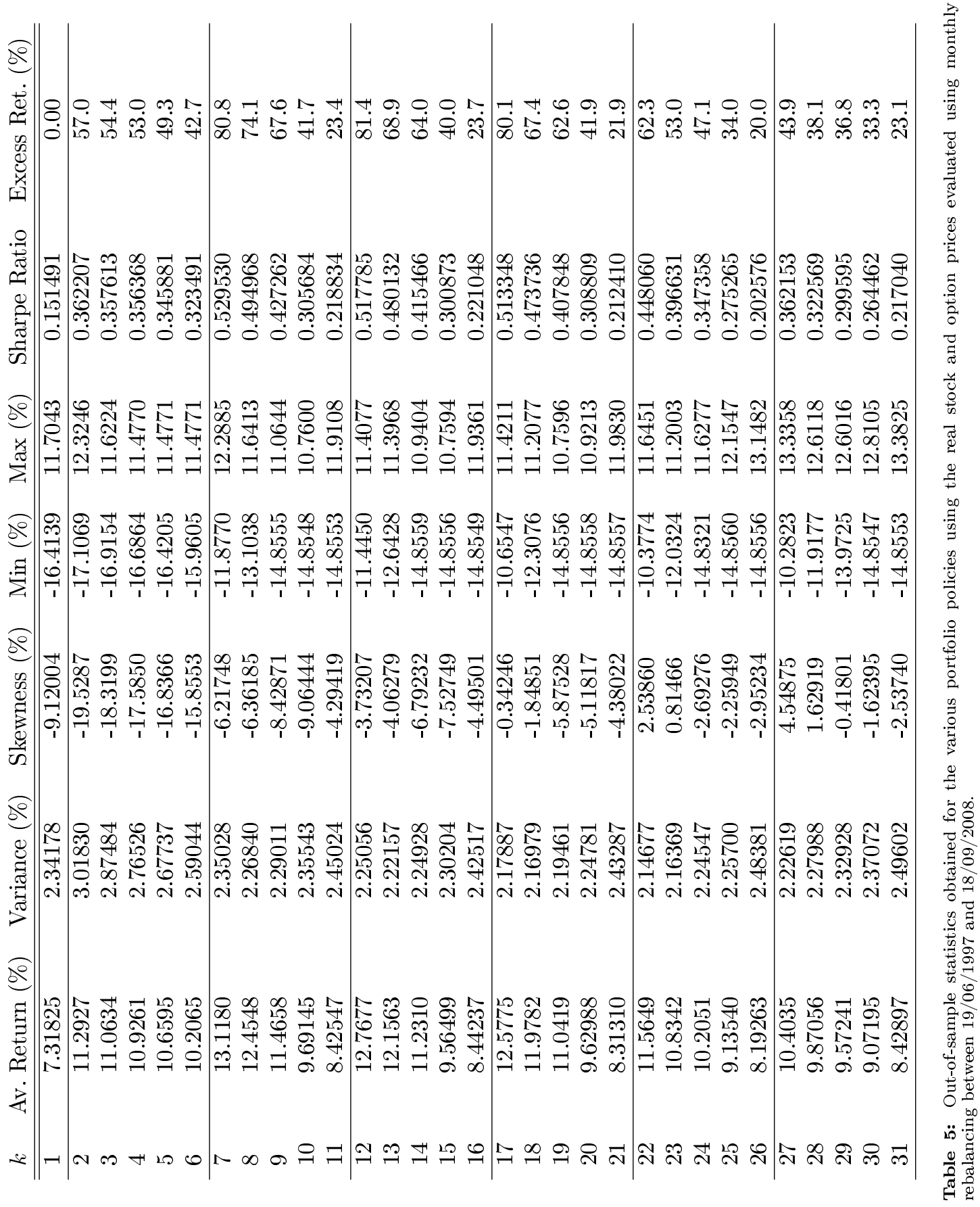

